ARTICLE

\title{
Arf6 controls retromer traffic and intracellular cholesterol distribution via a phosphoinositide-based mechanism
}

Catherine Marquer ${ }^{1}$, Huasong Tian ${ }^{1}{ }^{\dagger}$, Julie $\mathrm{Yi}^{1}$, Jayson Bastien ${ }^{1}{ }^{\dagger}$, Claudia Dall'Armi $^{1, \dagger}$, Young Joo Yang-Klingler ${ }^{1}$, Bowen Zhou ${ }^{1}$, Robin Barry Chan ${ }^{1} \&$ Gilbert Di Paolo, ${ }^{1, \dagger}$

Small GTPases play a critical role in membrane traffic. Among them, Arf6 mediates transport to and from the plasma membrane, as well as phosphoinositide signalling and cholesterol homeostasis. Here we delineate the molecular basis for the link between Arf6 and cholesterol homeostasis using an inducible knockout (KO) model of mouse embryonic fibroblasts (MEFs). We find that accumulation of free cholesterol in the late endosomes/lysosomes of Arf6 KO MEFs results from mistrafficking of Niemann-Pick type C protein NPC2, a cargo of the cation-independent mannose-6-phosphate receptor ( $\mathrm{Cl}-\mathrm{M} 6 \mathrm{PR})$. This is caused by a selective increase in an endosomal pool of phosphatidylinositol-4-phosphate (PI4P) and a perturbation of retromer, which controls the retrograde transport of $\mathrm{Cl}-\mathrm{M} 6 \mathrm{PR}$ via sorting nexins, including the PI4P effector SNX6. Finally, reducing PI4P levels in KO MEFs through independent mechanisms rescues aberrant retromer tubulation and cholesterol mistrafficking. Our study highlights a phosphoinositide-based mechanism for control of cholesterol distribution via retromer.

\footnotetext{
${ }^{1}$ Department of Pathology and Cell Biology, Columbia University Medical Center, New York City, New York 10032, USA. † Present addresses: Department of Pathology and Laboratory Medicine, Weill Cornell Medical College, New York City, New York 10021, USA (H.T.); Laura \& Isaac Perlmutter Cancer Center, NYU Langone Medical Center, New York City, New York 10016, USA (J.B.); IRBM Science Park S.P.A, 00071 Pomezia, Italy (C.D.); Denali Therapeutics, South San Francisco, California 94080, USA (G.D.P.). Correspondence and requests for materials should be addressed to C.M. (e-mail: cm2344@cumc.columbia.edu) or to G.D.P. (e-mail: gd2175@cumc.columbia.edu).
} 
ntracellular transport routes are under strict regulatory control in eukaryotic cells to ensure proper sorting of cargoes, maintain organelle identity and ultimately guarantee cell homeostasis. Among the key regulators of intracellular trafficking pathways, small GTPases, such as ADP ribosylation factor (Arf) family members, play a fundamental role in a compartmentspecific manner. Similar to other GTPases, Arf proteins cycle between an inactive GDP-bound form and an active GTP-bound form ${ }^{1}$. Unlike the other Arf family members (that is, Arf1-5), Arf6 is uniquely localized to the plasma membrane and to endosomes ${ }^{2,3}$, where it influences membrane trafficking. The role of Arf6 in various clathrin-dependent and -independent endocytic pathways as well as in recycling to the plasma membrane has been extensively studied ${ }^{4,5}$. A role for Arf6 in multivesicular body formation has also been recently described ${ }^{6}$. In addition, Arf6 regulates actin remodeling in such contexts as cell spreading, migration, cytokinesis, phagocytosis and neurite outgrowth $^{5,7}$. In vivo, Arf6 ablation is embryonically lethal in the mouse $^{8}$ but a conditional knockout (KO) model revealed a non-cell autonomous role for neuronal Arf6 in oligodendrocyte precursor cell migration and myelination ${ }^{9}$.

One of the major mechanisms of action of Arf6 occurs through the control of lipid metabolism. Indeed, Arf6 binds and activates phosphatidylinositol-4-phosphate 5-kinases (PI4P5Ks), also known as type I PIPKs (PIPKIs), which phosphorylate PI4P into $\mathrm{PI}(4,5) \mathrm{P}_{2}$ (ref. 10,11). In addition, Arf6 can activate phospholipase D (PLD) ${ }^{12}$, whose product phosphatidic acid can in turn activate PIPKIs (ref. 13). Overexpressing a constitutively active mutant of Arf6 (Arf6 Q67L) also results in enlarged endosomes that contain high levels of $\mathrm{PI}(4,5) \mathrm{P}_{2}$ (ref. 14). More recently, Arf6 has been implicated in the regulation of cellular cholesterol distribution. In cultured cells, most cholesterol is derived from cholesteryl ester-rich LDL particles present in the media. LDL-particles are internalized by the LDL receptor (LDLR) and trafficked to the lumen of late endosomes/ lysosomes (LE/LYS). Cholesteryl esters are first hydrolysed by lysosomal acid lipase to free cholesterol, which is believed to be transferred by NPC2, a small soluble protein of the LE/LYS lumen, to the polytopic membrane protein NPC1. The latter mediates the egress of cholesterol from the endolysosomal system, allowing for its distribution to other cellular compartments and inhibition of cholesterol biosynthesis via the cholesterol-sensing machinery operating in the endoplasmic reticulum ${ }^{15,16}$. Perturbation of cholesterol traffic through mutations of NPC1 or NPC2 genes causes Niemann-Pick Type C (NPC) disease, a fatal neurodegenerative disorder associated with accumulation of free cholesterol and other lipids in the endolysosomal system ${ }^{17}$. Several studies provided hints that Arf6 is involved in the control of cholesterol homeostasis. First, Arf6 Q67L enlarged endosomes contain high levels of free cholesterol, labelled with filipin ${ }^{18}$. Second, silencing Arf6 was found to increase cellular cholesterol content in HeLa cells, reminiscent of an NPC disease phenotype $^{19}$. In addition, Arf6 and components of its interactome were identified as 'hits' in a transcriptomic screen performed after cholesterol levels of cultured rat neurons were acutely increased ${ }^{20}$. More recently, Arf6 was a hit in a genomewide RNA-interference screen designed to identify genes required for intracellular transport of LDL-derived cholesterol, although this link was not explored further ${ }^{21}$. While these studies point to a link between Arf6 and cholesterol metabolism, the molecular basis underlying this relationship is unknown.

To address this fundamental question, we developed a novel model of Arf6 conditional KO in immortalized mouse embryonic fibroblasts (MEFs). We found that Arf6 depletion leads to cholesterol redistribution and accumulation in LE/LYS, a phenotype due to the mistargeting of NPC2 away from lysosomes. Because NPC2 is a cargo of the mannose-6-phosphate receptor $(\mathrm{M} 6 \mathrm{PR})^{22}$, we examined the function of retromer, which regulates the retrograde transport of the M6PR. We found that retromer function is impaired in Arf6 KO cells, thus leading to mislocalization of the cation-independent M6PR (CI)-M6PR away from the trans-Golgi network (TGN). We also found that PI4P levels were increased in Arf6 KO MEFs and that retromerpositive aberrant tubules emerged in part from PI4P-containing endosomes. Finally, we demonstrated that restoring PI4P levels rescues aberrant retromer tubules and cholesterol accumulation. This work therefore highlights a novel role for Arf6 in the regulation of retromer with critical implications for PI4P and cholesterol metabolism.

\section{Results}

Cholesterol redistributes to LE/LYS in Arf6 KO cells. To control the ablation of Arf6 acutely and inducibly, MEFs were generated from $A r f 6^{\text {Flox/Flox }}$ embryos also expressing a fusion of Cre recombinase with the ligand-binding domain of the oestrogen receptor (Cre-ER; Supplementary Fig. 1a). After immortalization, MEFs treated with tamoxifen showed gradually reduced levels of Arf6 protein by western blotting, leading to a complete absence of protein after 6 days (Fig. 1a; Supplementary Fig. 1b). We then assessed the impact of Arf6 ablation on cholesterol. Cholesterol content was first analysed by liquid chromatography-mass spectrometry (LC-MS) and was similar in Arf6 WT and KO MEFs (Fig. 1b). MEFs were subsequently stained with filipin, a fluorescent probe for free cholesterol (Fig. 1c). The overall filipin intensity was comparable in the two genotypes (Fig. 1d), in agreement with the LC-MS results. However, while wild-type (WT) cells exhibited a diffuse staining at the plasma membrane and the cytoplasm with higher fluorescence intensity in the perinuclear region, the pattern of filipin staining was altered in KO MEFs, where it accumulated in cytoplasmic puncta (Fig. 1c). Quantification of filipin fluorescence revealed there were more cholesterol puncta in Arf6 KO cells compared with controls (Fig. 1e). Filipin puncta in Arf6 KO MEFs also appeared significantly larger than in the controls (Fig. 1e). Aberrant filipin distribution could be rescued by expressing ARF6-HA (ref. 3) in Arf6 KO cells (Supplementary Fig. 2), indicating that the cholesterol redistribution phenotype is a direct and specific consequence of Arf6 ablation in those cells. We then identified the specific compartment where free cholesterol accumulated in Arf6 KO cells. Filipin accumulated in Rab7- and LAMP1-positive organelles but not in EEA1-positive organelles (Fig. 1f), indicating that cholesterol is redistributed to LE/LYS but not to early endosomes in KO cells. LAMP1 protein levels (Supplementary Fig. 3a) as well as the number and size of LAMP1-positive organelles (Supplementary Fig. 3b) were similar in both genotypes, indicating that Arf6 depletion did not cause any major structural disruption of LE/LYS.

NPC2 mistrafficking causes cholesterol redistribution. Since the accumulation of cholesterol in LE/LYS is reminiscent of NPC cells $^{17}$, we assessed whether the localization of NPC1 and/or NPC2 is affected by Arf6 depletion. As probed by Western blot, NPC1 protein levels were comparable $(P>0.05$, Student's $t$-test $)$ in Arf6 WT $(100 \pm 9 \%, \pm$ indicates s.e.m., $n=3)$ and Arf6 KO cells $(75 \pm 20 \%, n=3$; Supplementary Fig. 3c). NPC1-GFP was found on the membrane of LE/LYS in both Arf6 WT and $\mathrm{KO}$ cells and displayed extensive co-localization with LAMP1, independently of Arf6 presence (Supplementary Fig. 3d), suggesting that it is correctly trafficked in Arf6 KO cells. In contrast, co-localization of endogenous NPC2 and LAMP1 was greatly reduced in Arf6 KO cells compared with controls (Fig. 2a), 
although NPC2 protein levels were comparable in both genotypes (Fig. 2b; Arf6 WT $100 \pm 6 \%$ and Arf6 KO cells $110 \pm 26 \%, \pm$ indicates s.e.m., $n=3$ ). The reduced presence of
NPC2 in the LE/LYS compartment coincided with greater co-localization of this protein with Golgin97, a TGN marker (Supplementary Fig. 4), leading to the hypothesis that a

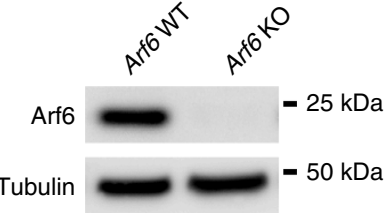

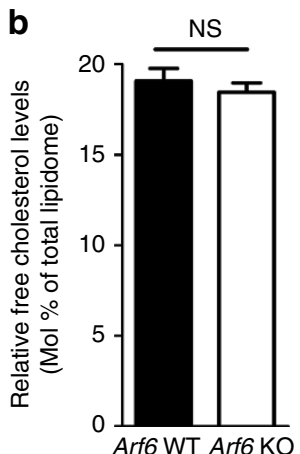

C
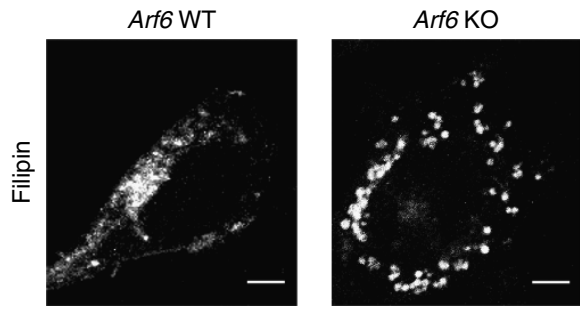
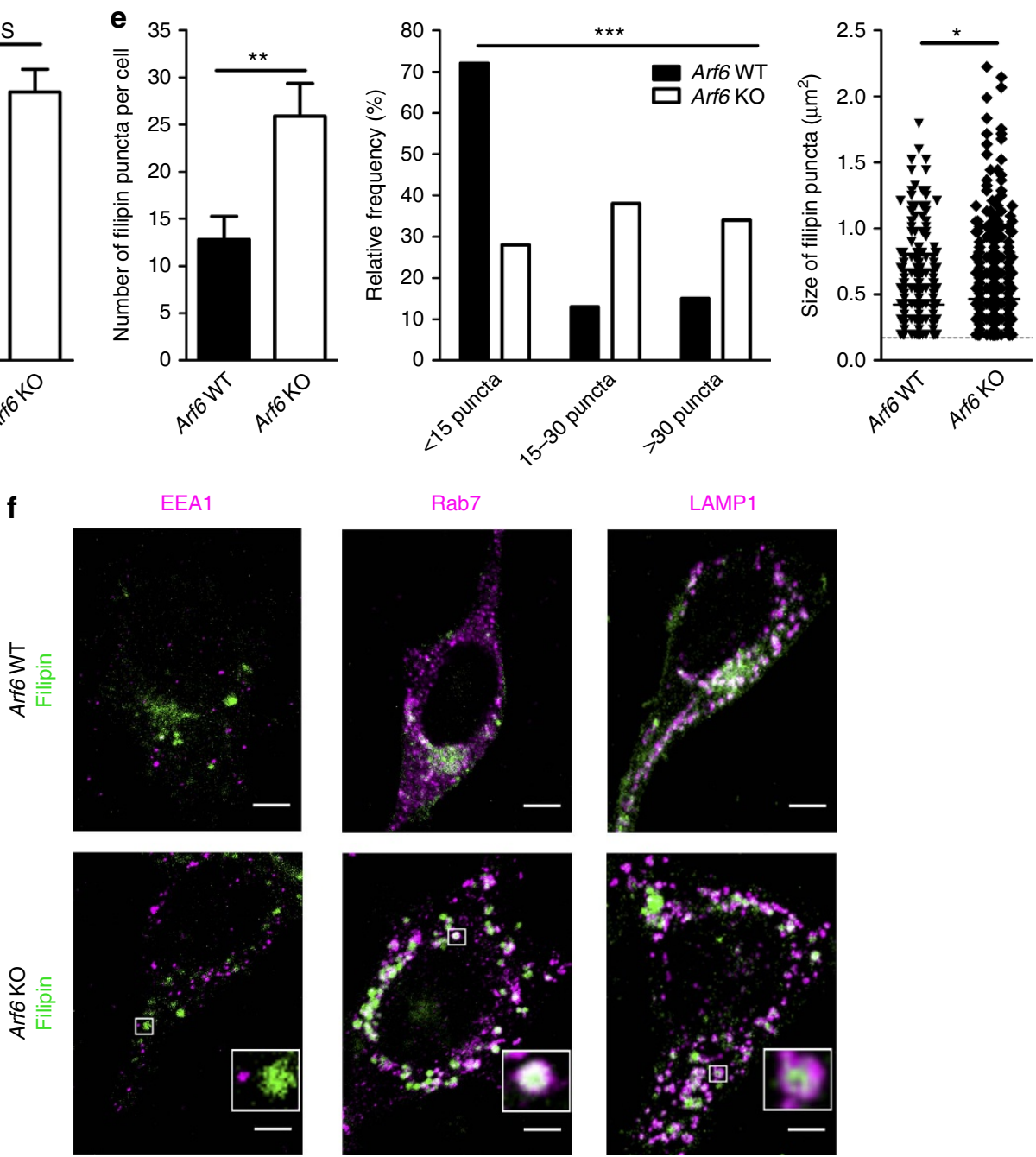

Figure 1 | Cholesterol is redistributed to late endosomes/lysosomes in Arf6 KO MEFs. (a) Western blot analysis of endogenous Arf6 levels in Arf6Flox/Flox; Cre-ER MEFs treated with DMSO (Arf6 WT) or tamoxifen (Arf6 KO). Tubulin was used as an equal loading marker. (b) Cholesterol levels measured by LC-MS were similar in Arf6 WT (19.1 \pm 0.7 molar $\%$ of total lipidome, \pm indicates s.e.m., $n=11)$ and KO cells (18.5 \pm 0.5 molar $\%$ of total lipidome, $n=12$ ). NS denotes $P>0.05$ in Student's $t$-test. (c) Representative confocal images of Arf6 WT and KO MEFs stained with filipin. Scale bar, $5 \mu$ m. (d) Normalized integrated densities of filipin staining were similar in Arf6 WT (100 $\pm 8 \%$, \pm indicates s.e.m., $\mathrm{n}=32$ cells from three experiments) and Arf6 KO cells ( $98 \pm 8 \%, n=42$ cells from four experiments). NS denotes $P>0.05$ in Student's $t$-test. (e) Quantification of filipin puncta in Arf6 WT and KO cells. Left panel, number of puncta per cell in Arf6 WT ( $13 \pm 2$ puncta per cell, \pm indicates s.e.m., $n=39$ cells from 3 experiments) and Arf6 KO cells ( $26 \pm 3$ puncta per cell, $n=29$ cells from 3 experiments). ${ }^{\star} P<0.01$ in Student's $t$-test. Middle panel, frequency distribution of the number of filipin puncta per cell. ${ }^{\star \star \star} P<0.001$ in $\chi^{2}$-test. Right panel, scatter dot blot of the size of filipin puncta in Arf6 WT $\left(0.42 \pm 0.01 \mu \mathrm{m}^{2}, \pm\right.$ indicates s.e.m., 498 puncta from 39 cells $)$ and $\mathrm{KO}$ cells $\left(0.47 \pm 0.01 \mu \mathrm{m}^{2}, 749\right.$ puncta from 29 cells). ${ }^{\star} P<0.05$ in Mann-Whitney test. (f) Representative immunostainings for EEA1, Rab7 or LAMP1 (magenta) of Arf6 WT or KO cells labelled with filipin (green). Scale bar, $5 \mu \mathrm{m}$. NS, not significant. 
a

LAMP1
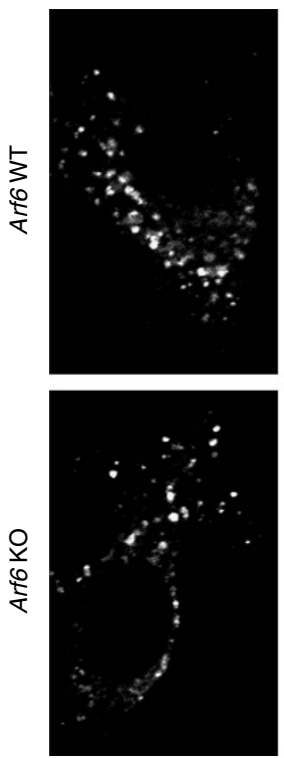

NPC2
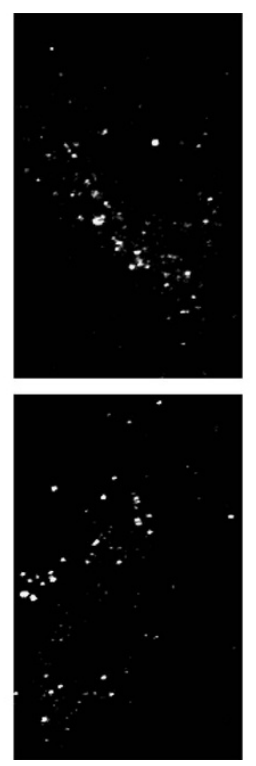
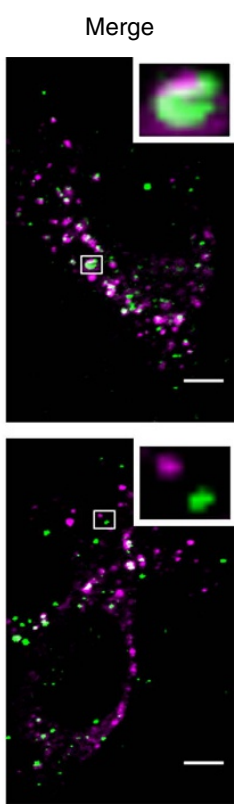

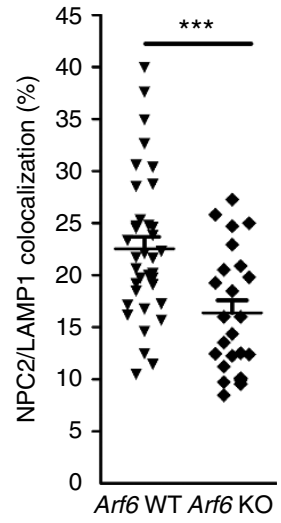

b

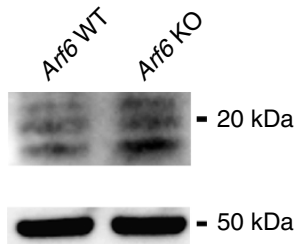

C

Vehicle
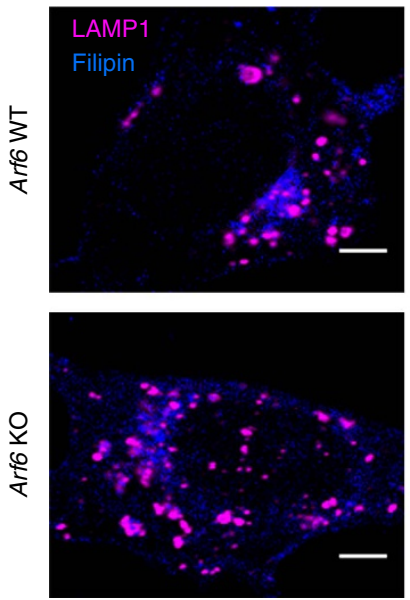

NPC2-Alexa488
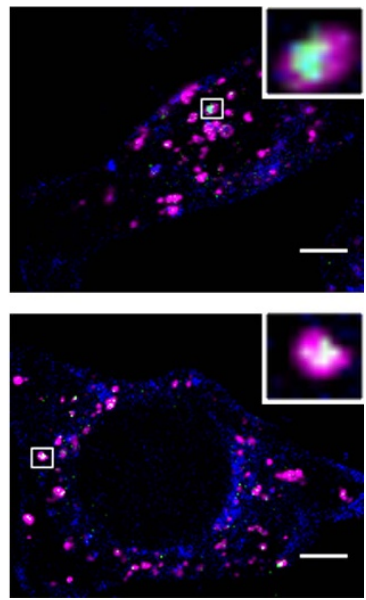
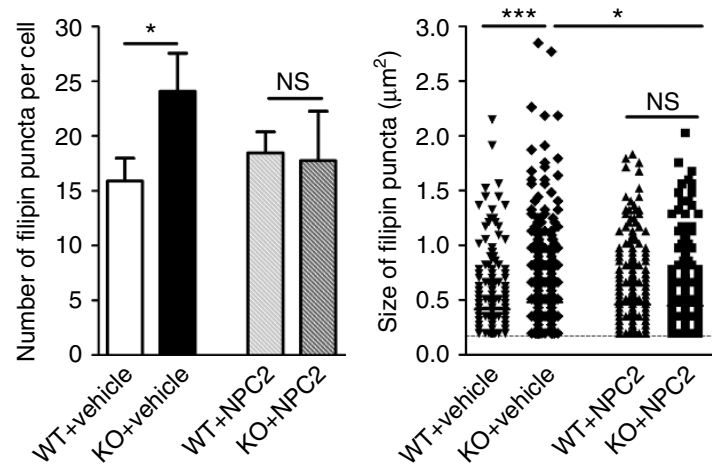

Figure 2 | NPC2 mistrafficking causes cholesterol redistribution in Arf6 KO MEFs. (a) Left panel, representative confocal images of Arf6 WT and KO cells immunostained for endogenous LAMP1 (magenta) and NPC2 (green). Scale bar, $5 \mu \mathrm{m}$. Right panel, NPC2/LAMP1 co-localization levels in Arf6 WT ( $23 \pm 1 \%, \pm$ indicates s.e.m., $n=36$ cells from three experiments) and Arf6 KO cells $\left(16 \pm 1 \%, n=24\right.$ cells from three experiments). ${ }^{\star \star *} P<0.001$ in Student's t-test. (b) Western blot analysis of endogenous NPC2 levels in Arf6 WT and KO cells. Tubulin was used as an equal loading marker. NPC2 protein levels were similar ( $P>0.05$ in Student's $t$-test) in Arf6 WT (100 $\pm 6 \%, \pm$ indicates s.e.m., $n=3)$ and KO cells $(110 \pm 26 \%, n=3)$. (c) NPC2-Alexa488 (green) or vehicle was added to the culture media of Arf6 WT and KO cells for $24 \mathrm{~h}$. Cells were then washed, fixed and stained for endogenous LAMP1 (magenta) and cholesterol (with filipin, blue). Left panel, representative confocal images. Scale bar, $5 \mu \mathrm{m}$. Inset shows that exogenous NPC2 reaches the LAMP1 compartment. Center and right panel, quantification of filipin puncta. Center, number of filipin puncta in vehicle-treated Arf6 WT (16 \pm 2 puncta per cell, \pm indicates s.e.m., $n=34$ cells, three experiments), vehicle-treated KO ( $24 \pm 3$ puncta per cell, $n=35$ cells, three experiments), NPC2-treated Arf6 WT (18 \pm 2 puncta per cell, $n=36$ cells, three experiments) and NPC2-treated KO cells ( $18 \pm 5$ puncta per cell, $n=28$ cells, three experiments). NS and * stand for $P>0.05$ and $P<0.05$, respectively, in $t$-test with Welch's correction. Right, scatter dot blot of the size of filipin puncta of vehicle-treated Arf6 WT $\left(0.41 \pm 0.01 \mu \mathrm{m}^{2}, \pm\right.$ indicates s.e.m., 540 puncta from 34 cells $)$, vehicle-treated Arf6 KO $\left(0.48 \pm 0.01 \mu \mathrm{m}^{2}, 843\right.$ puncta from 35 cells $)$, NPC2-treated Arf6 WT $\left(0.46 \pm 0.01 \mu \mathrm{m}^{2}, 663\right.$ puncta from 36 cells $)$ and NPC2-treated Arf6 KO cells $\left(0.45 \pm 0.01 \mu \mathrm{m}^{2}, 496\right.$ puncta from 28 cells $)$. NS denote $P>0.05$, ${ }^{\star} P<0.05$ and ${ }^{\star \star \star} P<0.001$ in Mann-Whitney test, respectively. NS, not significant.

mistargeting of NPC2 and its thus reduced activity may be responsible for cholesterol accumulation in LE/LYS. To test this, we bypassed the anterograde pathway for delivery of NPC2 to endosomes by exogenously adding fluorescent NPC2-Alexa488 to MEFs and monitored cholesterol distribution with filipin (Fig. 2c). In vehicle-treated KO cells, cholesterol accumulated in LE/LYS as expected (Fig. 2c). Exogenous NPC2 was able to be internalized and reach the LAMP1-positive LE/LYS compartment and, remarkably, decreased both the number and size of filipin puncta in the Arf6 KO cells (Fig. 2c), corroborating our hypothesis that Arf6 depletion induces cholesterol redistribution to LE/LYS through NPC2 mistrafficking.

Mislocalisation of CI-M6PR causes mistrafficking of NPC2. NPC2 trafficking was reported to be dependent on M6PRs, with a more significant contribution of CI-M6PR relative to cation-dependent-M6PR (ref. 22). CI-M6PR cycles between the TGN and endosomes, where it transports its ligands, mostly 
soluble lysosomal proteins, including hydrolases. The latter are then released in endosomes as a result of a lower $\mathrm{pH}$ and passively delivered to the LE/LYS where their function is needed. The retrograde transport of CI-M6PR from endosomes to the TGN is mediated primarily by the retromer complex ${ }^{23,24}$. We thus investigated whether NPC2 mistrafficking resulted from deficits in the traffic of CI-M6PR. In Arf6 WT cells, CI-M6PR was highly concentrated in the perinuclear region, in accordance with previous reports that it primarily accumulates in the TGN at steady state ${ }^{25}$ (Fig. 3a). However, in Arf6 KO cells, CI-M6PR appeared more dispersed and was present not only in the TGN area but also on vesicular structures that were positive for $\mathrm{Vps} 35$, the core component of retromer (Fig. 3a).

The retromer complex function is impaired in Arf6 $\mathrm{KO}$ cells. We then asked whether CI-M6PR mislocalization was due to a deficit in its retrograde transport caused by a malfunction of the retromer. The retromer complex is composed of two subcomplexes. The cargo-selective core subcomplex, composed of Vps35, Vps29 and Vps26, is involved in cargo recognition and sorting. The membrane-deforming subcomplex, composed of a sorting nexin (SNX) heterodimer, is involved in tubulo-vesicular carrier formation from endosomal membranes ${ }^{26}$. In the case of CI-M6PR transport, the specific SNX isoforms involved are heterodimers of SNX1 or SNX2 and SNX5 or SNX6 (refs 23,27-30).

We thus examined the relationship between Arf6 and retromer using confocal microscopy. Because various antibodies failed to specifically recognize endogenous Arf6 in immunofluorescence applications, two different ARF6 constructs, ARF6-HA (ref. 3) and CyPET-ARF6 (ref. 31), were exogenously expressed in HeLa cells, which were then stained for VPS35. A pool of Arf6 and retromer were found on the same endosomal compartments, suggesting a potential functional interaction (Supplementary Fig. 5). We then assessed the potential impact of Arf6 deletion on retromer levels by focusing on the core subunit of retromer and found that Vps35 protein levels were similar in Arf6 WT and KO cells (Fig. 3b). However, the co-localization between Vps35 and EEA1, an early endosome marker, was increased in Arf6 KO cells, compared with WT cells (15\% versus 10\%, respectively, Fig. 3c,d), showing that retromer was partially redistributed to early endosomes. While the number of tubulo-vesicular Vps35-positive retromer carriers was comparable, their size was increased in Arf6 KO cells (Fig. 3e). In contrast, the number and size of EEA1positive early endosomes were similar in both genotypes (Fig. 3e). When visualized with super-resolution three-dimensional (3D)-Structured Illumination Microscopy (SIM), the size of retromer tubulo-vesicular carriers appeared larger in Arf6 $\mathrm{KO}$ cells, where Vps35 and EEA1 formed a continuous alveolarshaped endosomal network (Fig. 3f; Supplementary Movies 1-4).

To test for potential changes in the dynamics of retromer tubules, we transfected MEFs with GFP-SNX6 and imaged them by spinning-disk microscopy (Supplementary Movies 5 and 6). As shown in Fig. 3g, there were more GFP-SNX6-positive tubules in Arf6 KO cells than in controls, and these tubules were both longer and more persistent. SNX6 tubules were still able to move towards the perinuclear region in Arf6 KO cells (Supplementary Movies 6), indicating no gross malfunction of molecular motors/microtubules transport. Altogether, our data suggest that Arf6 ablation causes alterations in retromer tubule dynamics, which are consistent with fission defects.

Retromer knockdown causes cholesterol increase in LE/LYS. Our data suggest that Arf6 deletion causes a retromer defect which in turn leads to aberrant localization of CI-M6PR and its cargo NPC2 and finally to accumulation of cholesterol in LE/LYS. If this hypothesis is correct, then retromer deficiency should mimic the cholesterol phenotype observed in Arf6 KO cells. We thus treated HeLa cells with small interfering (siRNAs) to VPS35 and monitored the cholesterol distribution. After VPS35 siRNA transfection (VPS35 KD), VPS35 protein levels were decreased to $22 \pm 11 \%$ ( \pm indicates s.e.m., $n=3$ ) of those found in scramble siRNA-transfected cells, as assessed by western blot (Fig. 4a). Cells treated with siRNAs were fixed, immunostained for LAMP1 and VPS35 and labelled with filipin (Fig. 4b). Unlike Arf6 KO MEFs, VPS35 KD HeLa cells showed an overall increase in the levels of free cholesterol (Fig. 4c). However, similar to our observations in Arf6 KO cells, filipin accumulated primarily in large LAMP1-positive puncta (Fig. 4b). Quantification of filipin puncta showed that there was on average $\sim 77 \%$ more puncta/cell in VPS35 KD cells and that there was a significant shift in the relative distribution towards cells with a very large number of puncta (30 or more; Fig. 4d). Filipin puncta were also significantly larger in VPS35 KD cells than in controls (Fig. 4e). An increase in the number of filipin puncta was also observed when HeLa cells were treated with siRNAs targeting SNX1 and SNX2 or SNX5 and SNX6, the main sorting nexin isoforms involved in CI-M6PR trafficking ${ }^{23,27-30}$ (Supplementary Fig. 6). Taken together, these results show that depletion of retromer causes an increase in cholesterol levels and an accumulation of cholesterol in the LE/LYS compartment, consistent with our hypothesis. The retromer $\mathrm{KD}$ phenotype appears to be slightly more marked than the Arf6 KO phenotype vis-à-vis global cholesterol accumulation, perhaps reflecting the impact of a full versus a partial loss of function of retromer in VPS35 KD HeLa cells and Arf6 KO MEFs, respectively.

PI4P accumulates on retromer-positive early endosomes. To further delineate the molecular mechanisms underlying the retromer defect in Arf6 KO cells, we attempted to identify the Arf6 effector responsible for this phenotype. As stated earlier, some of the key effectors of Arf6 are PIPKIs, which catalyse the phosphorylation of PI4P into $\mathrm{PI}(4,5) \mathrm{P}_{2}$ (refs 10,11). We thus analysed the anionic phospholipid content of MEFs by HPLC combined with suppressed conductivity. Interestingly, we observed a $\sim 20 \%$ increase in PI4P in Arf6 KO cells, with no changes in phosphatidylserine, cardiolipin, PI or, more intriguingly, $\mathrm{PI}(4,5) \mathrm{P}_{2}$ (Fig. 5a). As it was reported that phosphatidic acid levels could also play a role in PIPKIs regulation ${ }^{13}$, we measured phosphatidic acid levels by HPLC and LC-MS. Phosphatidic acid levels measured by HPLC were similar $(P>0.05$, Student's $t$-test) in Arf6 WT (100 \pm 3 normalized molar \% of measured lipids, \pm indicates s.e.m., $n=3)$ and Arf6 $\mathrm{KO}$ cells (108 \pm 16 normalized molar \% of measured lipids, $n=3)$. Similarly, phosphatidic acid levels measured by LC-MS were comparable $(P>0.05$, Student's $t$-test $)$ in Arf6 WT $(0.077 \pm 0.010$ molar $\%$ of measured lipids, \pm indicates s.e.m., $n=11)$ and Arf6 KO cells (0.064 \pm 0.007 molar \% of measured lipids, $n=12$ ), making an indirect contribution of PLD unlikely.

Further investigation of PI4P levels by immunofluorescence also revealed a striking approximately twofold increase in PI4P fluorescence in Arf6 KO MEFs relative to controls (Fig. 5b). As expected $^{32}$, a large pool of PI4P co-localized with the TGN marker Golgin97 in both genotypes (Fig. 5b). However, as recently reported by others ${ }^{33}$, PI4P was also present on more peripheral compartments likely corresponding to endosomes (Fig. 5b). We thus tested whether some of these PI4P-positive compartments are early endosomes by co-immunostaining MEFs for PI4P and EEA1 and found that the co-localization between PI4P and EEA1 was increased by twofold in Arf6 KO cells 
compared with controls (Fig. 5c). PI4P thus markedly accumulates on early endosomes. We also assessed whether PI4P accumulates on endosomal pools where retromer is present by immunostaining MEFs for endogenous PI4P and SNX6. We found not only that the overall PI4P/SNX6 co-localization was increased in Arf6 KO cells compared with WT cells (Fig. 5d, right panel) but also that the cellular region where this co-localization took place was different (Fig. 5d, left panel). While in Arf6 WT cells, PI4P and SNX6 mostly co-localized in the perinuclear region, they also co-localized in more peripheral compartments in Arf6 KO cells (Fig. 5d, insets). Comparable results were obtained when immunostaining MEFs for endogenous PI4P and retromer a
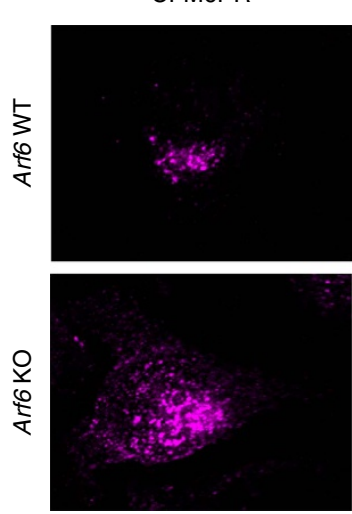

C
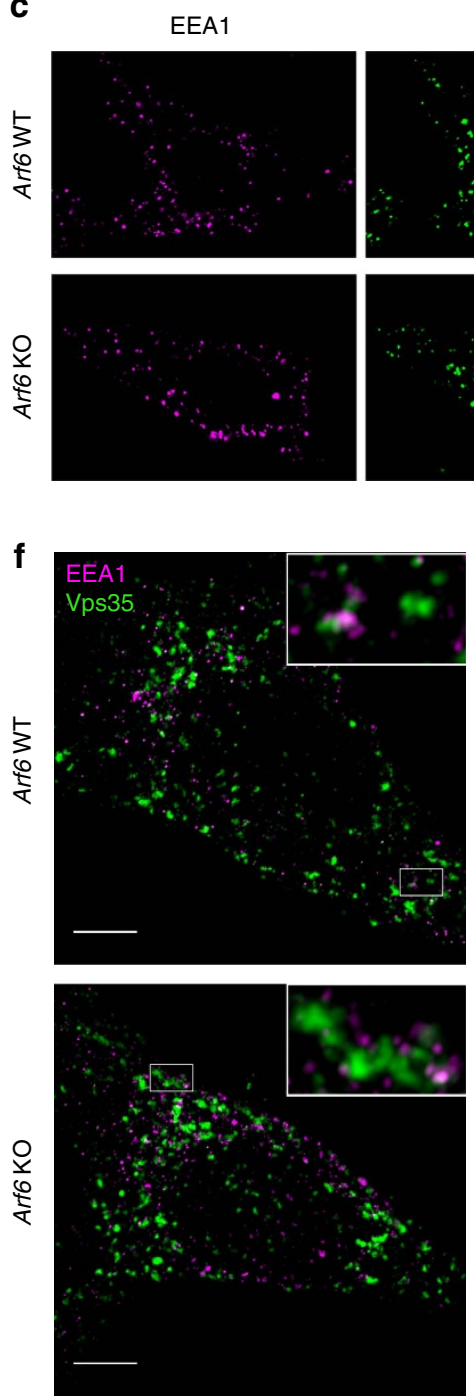

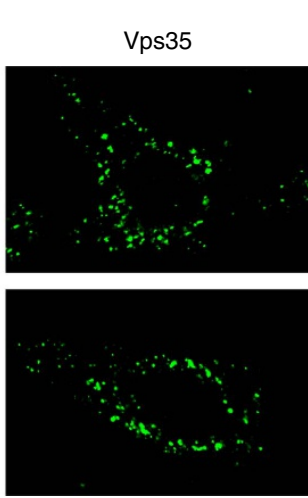

Vps35
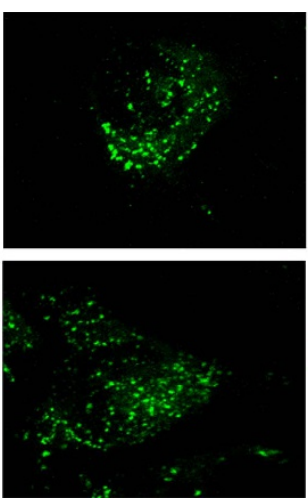

g
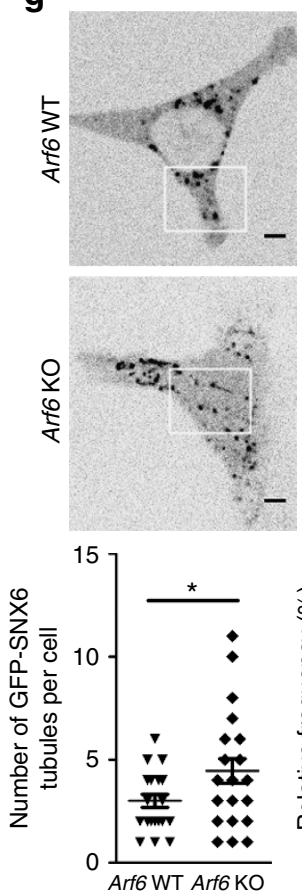

Merge
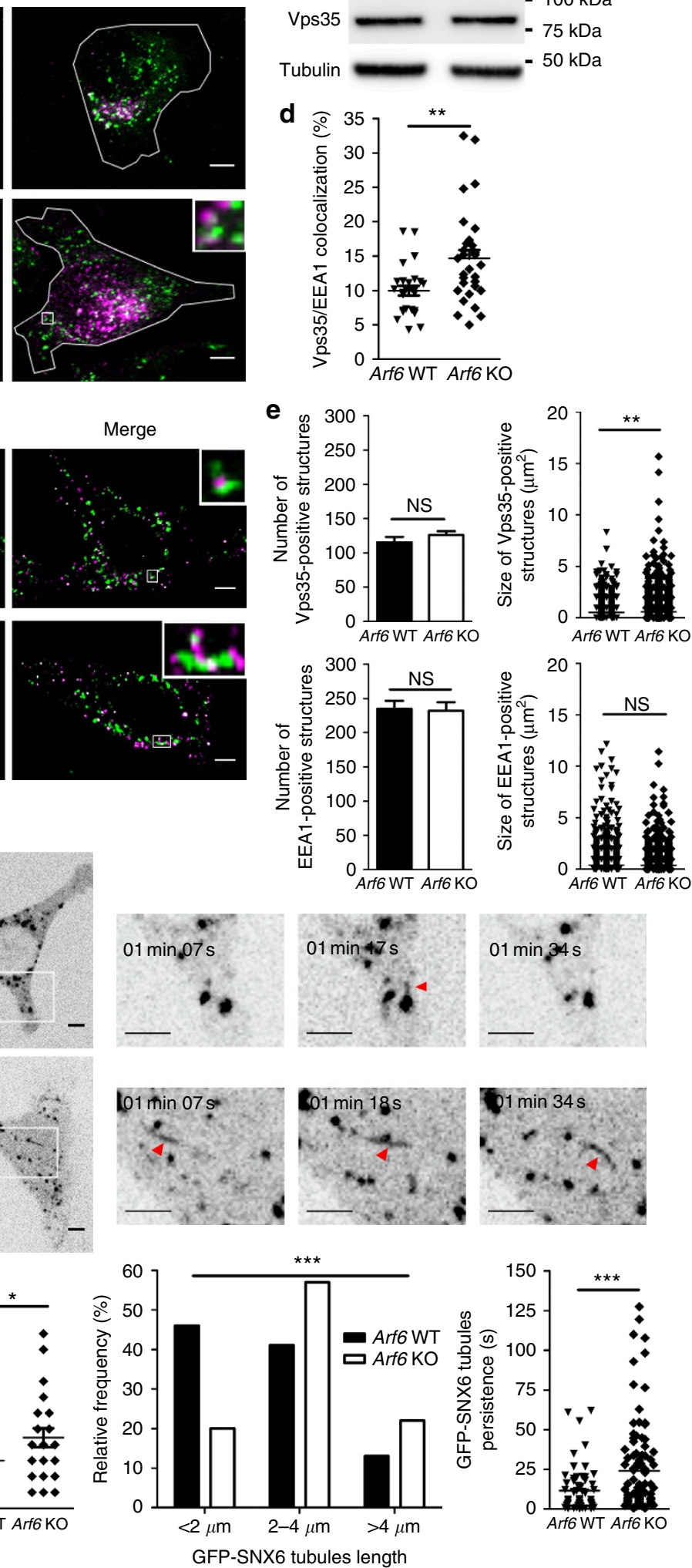
a

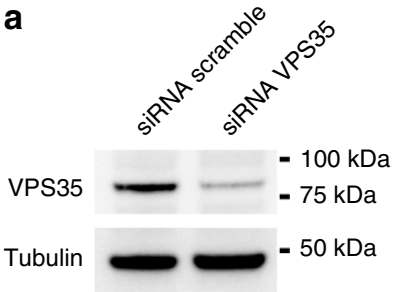

b

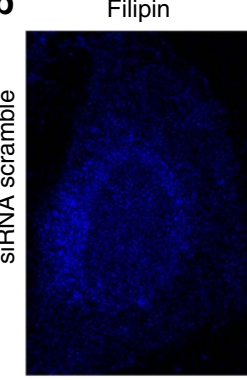

LAMP1
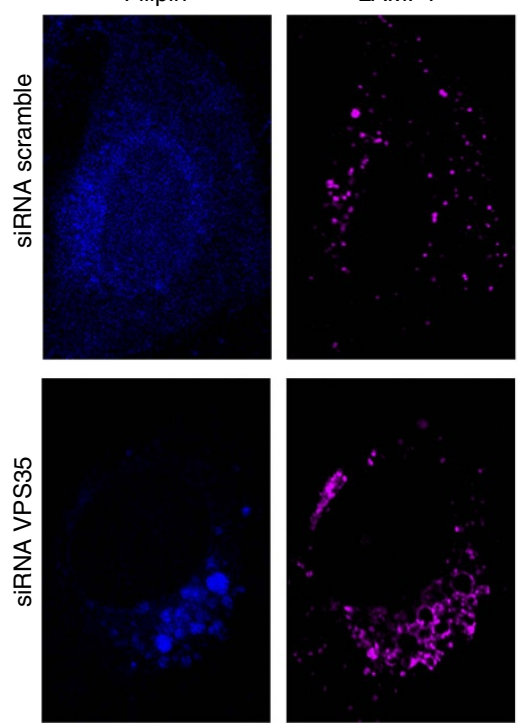

Vps35
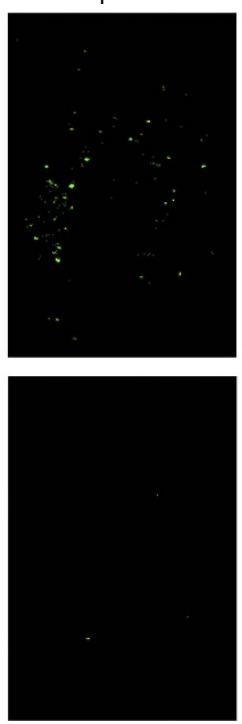

Merge
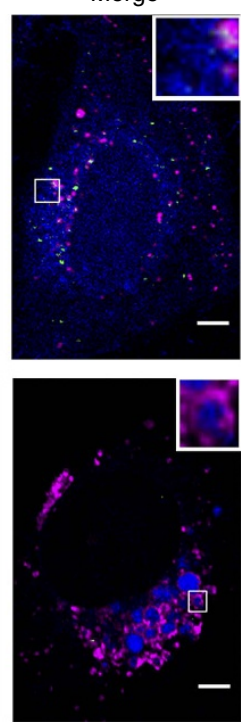
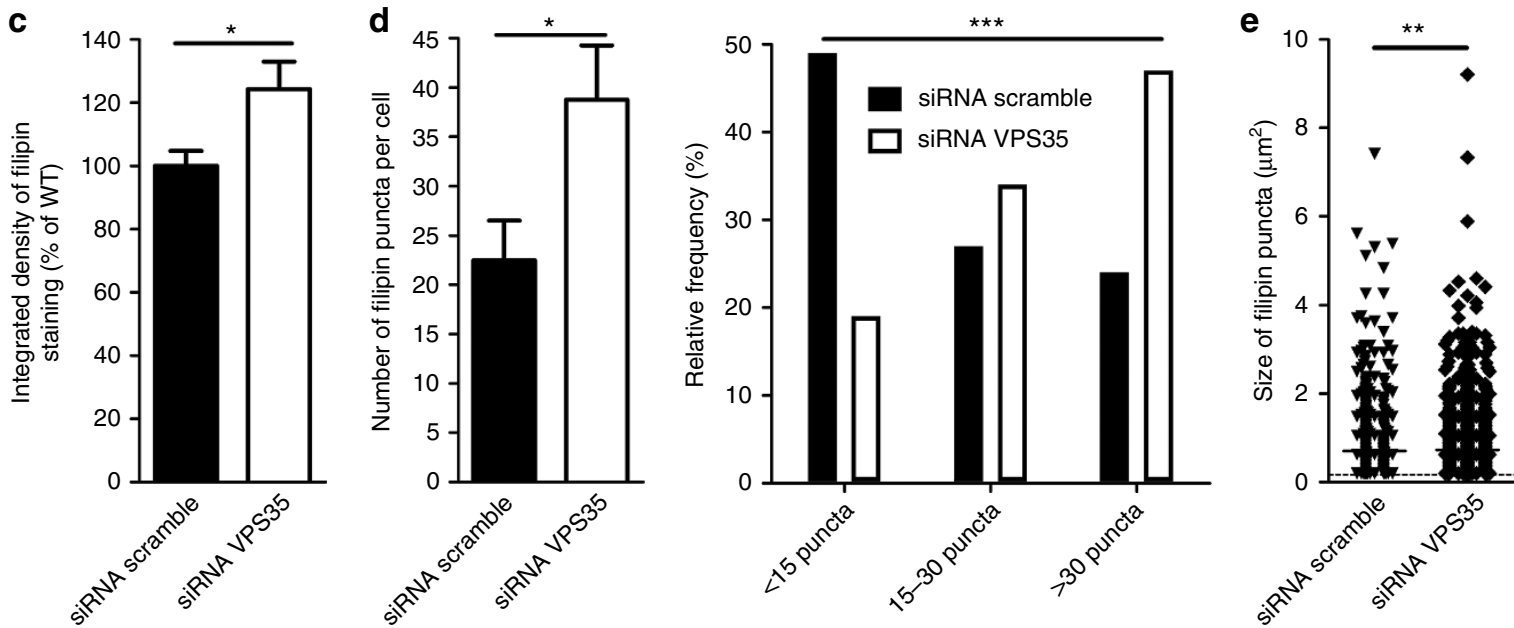

Figure 4 | Cholesterol is redistributed to late endosomes/lysosomes in VPS35 KD HeLa cells. (a) Western blot analysis of VPS35 levels in HeLa cells transfected with scramble or VPS35 siRNA. Tubulin was used as an equal loading marker. (b) Representative confocal images of HeLa cells transfected with scramble or VPS35 siRNA, immunostained for LAMP1 (magenta) and VPS35 (green) and labelled with filipin (blue). Scale bar, $5 \mu$ m. (c) Normalized integrated densities of filipin staining in scramble (100 $\pm 5 \%, \pm$ indicates s.e.m., $n=29$ cells, three experiments) and VPS35 siRNA cells ( $124 \pm 9 \%, n=28$ cells, three experiments). ${ }^{\star} P<0.05$ in $t$-test with Welch's correction. (d) Quantification (left) and frequency distribution (right) of the number of filipin puncta in scramble ( $22 \pm 4$ puncta per cell, \pm indicates s.e.m., $n=33$ cells, three experiments) and VPS35 siRNA cells ( $39 \pm 5$ puncta per cell, $n=32$ cells, three experiments). ${ }^{\star} P<0.05$ in Student's $t$-test and ${ }^{\star \star \star} P<0.001$ in $\chi^{2}$-test. (e) Scatter dot blot of the size of filipin puncta in scramble $\left(0.71 \pm 0.03 \mu m^{2}\right.$, \pm indicates s.e.m., 741 puncta from 33 cells $)$ and VPS35 siRNA cells $\left(0.73 \pm 0.02 \mu \mathrm{m}^{2}, 1,241\right.$ puncta from 32 cells $)$. ${ }^{\star \star} P<0.01$ in Mann-Whitney test.

Figure 3 | Retromer function is impaired in Arf6 KO MEFs. (a) Representative maximum intensity projections of Arf6 WT and KO cells immunostained for $\mathrm{Cl}-M 6 P R$ (magenta) and Vps35 (green). Scale bar, $5 \mu \mathrm{m}$. (b) Western blot analysis of Vps35 levels in Arf6 WT $(100 \pm 5 \%, n=5)$ and KO cells $(113 \pm 5 \%$, $n=5), P>0.05$ in Student's $t$-test. Tubulin was used as an equal loading marker. (c) Representative confocal images of Arf6 WT and KO cells immunostained for EEA1 (magenta) and Vps35 (green). Scale bar, $5 \mu \mathrm{m}$. (d) Levels of Vps35/EEA1 co-localization in Arf6 WT (10 $\pm 1 \%, n=26$ cells) and $\mathrm{KO}$ cells $\left(15 \pm 1 \%, n=30\right.$ cells). ${ }^{\star \star} P<0.01$ in $t$-test with Welch's correction. (e) Top left panel, number of Vps35 puncta in Arf6 WT (116 \pm 8 puncta per cell, $n=28$ cells $)$ and KO cells ( $126 \pm 6$ puncta per cell, $n=28$ cells). ns stands for $P>0.05$ in Student's $t$-test. Top right, size of Vps35 puncta in WT $\left(0.53 \pm 0.01 \mu \mathrm{m}^{2}, 3,241\right.$ puncta) and KO cells $\left(0.59 \pm 0.01 \mu \mathrm{m}^{2}, 3,529\right.$ puncta). ${ }^{\star \star}$ stands for $P<0.01$ in Mann-Whitney test. Bottom left, number of EEA1 puncta in WT ( $235 \pm 12$ puncta per cell, $n=28$ cells) and KO cells ( $232 \pm 13$ puncta per cell, $n=23$ cells). Bottom right, size of EEA1 puncta in WT $\left(0.40 \pm 0.01 \mu \mathrm{m}^{2}, 6,571\right.$ puncta) and KO cells $\left(0.39 \pm 0.01 \mu \mathrm{m}^{2}, 5,334\right.$ puncta). ns stands for $P>0.05$ in Student's t-test (left) and Mann-Whitney test (right). (f) Maximum intensity projections of 3D SIM z-stacks of Arf6 WT and KO cells immunostained for EEA1 (magenta) and Vps35 (green). Scale bar, $5 \mu \mathrm{m}$. (g) Spinning-disk confocal imaging of Arf6 WT and KO MEFs expressing GFP-SNX6. Top panel, representative images. Scale bar, 5 and $1 \mu \mathrm{m}$ (insets). Arrows indicate tubules. Lower panel, quantification of GFP-SNX6 tubules. Left, number of GFP-SNX6 tubules in Arf6 WT ( $3 \pm 0.3$ tubules per cell, $n=21$ cells) and $\mathrm{KO}$ cells ( $4.5 \pm 0.6$ tubules per cell, $n=22$ cells). ${ }^{\star} P<0.05$ in $t$-test with Welch's correction. Center, frequency distribution of GFP-SNX6 tubules length in Arf6 WT $(2.6 \pm 0.2 \mu \mathrm{m}, n=63$ tubules $)$ and KO cells $(3.2 \pm 0.2 \mu \mathrm{m}, n=97$ tubules $) .{ }^{\star \star \star} P<0.001$ in $\chi^{2}$-test. Right, persistence of GFP-SNX6 tubules in Arf6 WT (11.6 $\pm 1.8 \mathrm{~s}, n=62$ tubules) and KO cells $\left(24.1 \pm 2.9 \mathrm{~s}, n=97\right.$ tubules). ${ }^{\star \star \star} P<0.001$ in $t$-test with Welch's correction. All values are given as mean \pm s.e.m. 
a

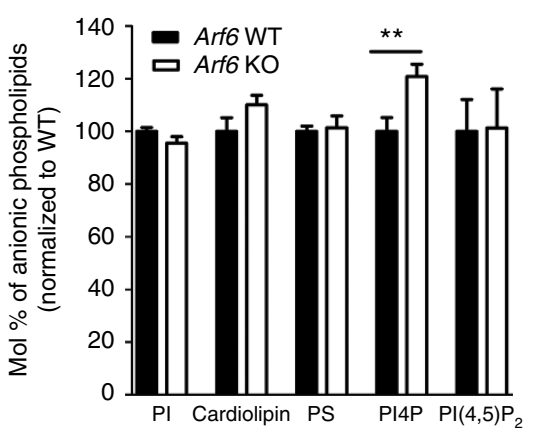

b

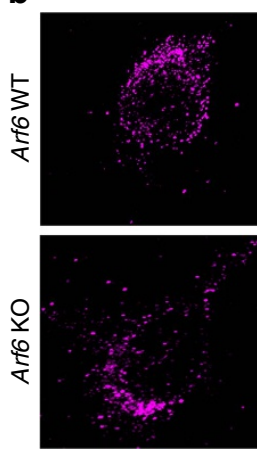

Golgin97
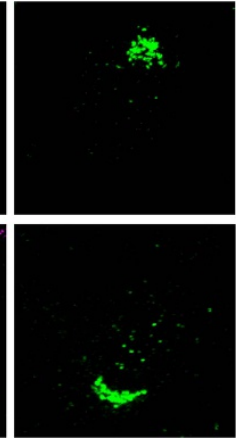
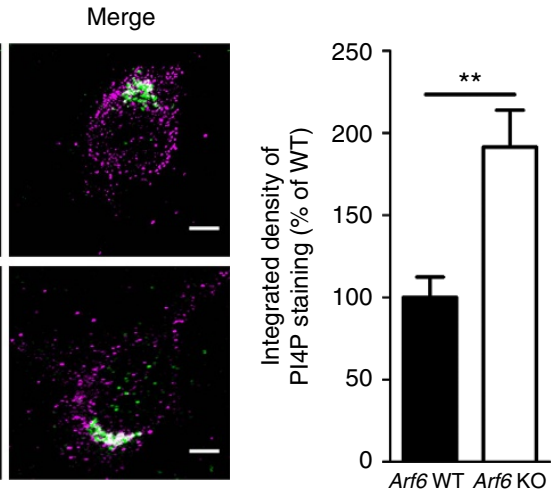
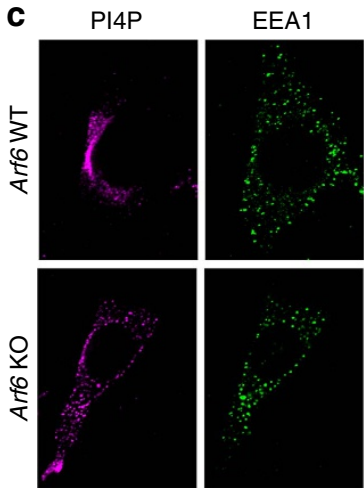

Merge

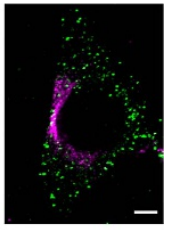

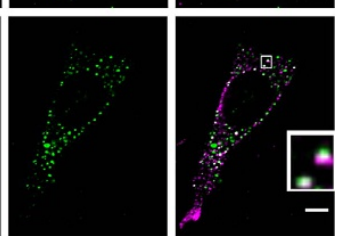

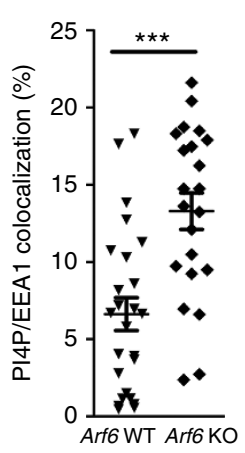

d
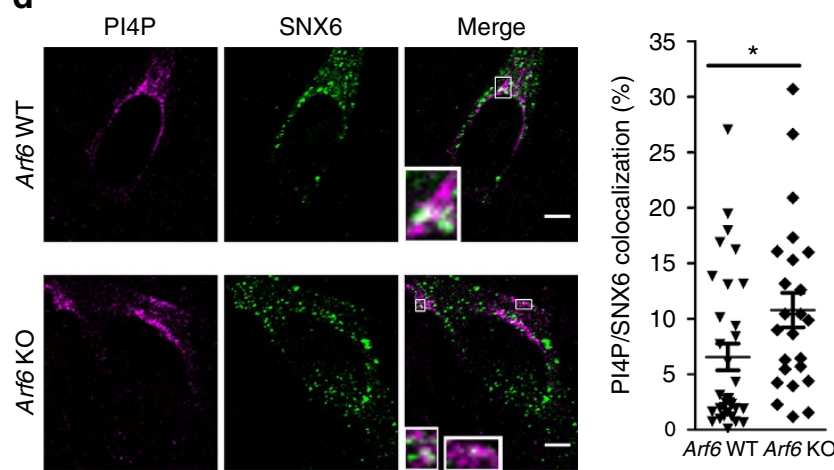

Figure 5 | PI4P is increased in Arf6 KO cells and accumulates in retromer-positive endosomes. (a) Bar diagram showing lipid levels in Arf6 WT and KO cells. Measurements were made by anionic exchange HPLC with suppressed conductivity detection, expressed in molar percentage of total anionic phospholipid measured and normalized to Arf6 WT levels. PI4P levels were increased in Arf6 KO cells ( $121 \pm 5 \%$, \pm indicates s.e.m., $n=9$ ) compared with controls $(100 \pm 5 \%, n=9) .{ }^{\star \star} P<0.01$ in Student's $t$-test. (b) Left, representative confocal images of Arf6 WT and KO cells immunostained for PI4P (magenta) and Golgin97 (green). Scale bar, $5 \mu \mathrm{m}$. Right, normalized integrated densities of PI4P staining were increased in Arf6 KO cells (191 $\pm 23 \%$, \pm indicates s.e.m., $n=27$ cells, three experiments) compared to controls ( $100 \pm 12 \%, n=22$ cells, three experiments). ${ }^{\star \star} P<0.01$ in $t$-test with Welch's correction. (c) Left, representative confocal images of Arf6 WT and KO cells immunostained for PI4P (magenta) and EEA1 (green). Scale bar, $5 \mu \mathrm{m}$. Right, PI4P/EEA1 co-localization levels in Arf6 WT ( $7 \pm 1 \%, \pm$ indicates s.e.m., $n=25$ cells, three experiments) and KO cells ( $13 \pm 1 \%, n=22$ cells, three experiments). ${ }^{\star \star \star} P<0.001$ in Student's $t$-test. (d) Left, representative confocal images of Arf6 WT and KO cells immunostained for PI4P (magenta) and SNX6 (green). Scale bar, $5 \mu \mathrm{m}$. Right, PI4P/SNX6 co-localization levels in WT ( $\pm \pm 1 \%, \pm$ indicates s.e.m., $n=33$ cells, three experiments) and KO cells $\left(11 \pm 2 \%, n=24\right.$ cells, three experiments). ${ }^{\star} P<0.05$ in Student's $t$-test.

core component Vps35 (Supplementary Fig. 7). We thus concluded that in Arf6 KO cells, both PI4P and the retromer complex accumulate on endosomes.

Lowering PI4P levels rescues retromer function. These data led us to postulate that endosomal PI4P accumulation may be responsible for the defect in retromer tubules dynamics observed in Arf6 KO cells (Fig. 3g). To test this, we used phenylarsine oxide (PAO), a PI4K inhibitor ${ }^{34}$, to lower the levels of PI4P. After $30 \mathrm{~min}$ of $1 \mu \mathrm{M}$ PAO treatment, levels of PI4P in Arf6 KO cells decreased by $\sim 20 \%$ and $\sim 35 \%$, as assessed by immunostaining and HPLC, respectively (Fig. 6a,b). We quantified the number of GFP-SNX6 tubules in cells treated with dimethyl sulfoxide (DMSO) or PAO and visualized by spinning-disk microscopy (Fig. 6c; Supplementary Movies 7-9). At time 0, both sets of cells had a similar number of tubules $(P>0.05, t$-test with Welch's correction). The number of GFP-SNX6 tubules in DMSO-treated Arf6 KO MEFs was unchanged with time $(P>0.05$, one-way analysis of variance (ANOVA)). In contrast, PAO-treated Arf6 KO cells showed a decreased number of tubules after $15 \mathrm{~min}$ of treatment $(40 \pm 12 \%$ of the number of tubules at time 0 , \pm indicates s.e.m.) and this decrease was even more pronounced after $30 \mathrm{~min}$ of treatment $(33 \pm 8 \%$ of the number of tubules at time 0). Decreasing the levels of PI4P can thus rescue the defect in retromer tubule dynamics.
Lowering PI4P levels rescues cholesterol redistribution. To test whether PI4P increase is also the cause of cholesterol accumulation in the LE/LYS of Arf6 KO cells, we assessed the impact of lowering PI4P levels on cholesterol localization using different but complementary approaches. First, we used PAO treatment and labelled cells for PI4P and filipin (Fig. 6d). After $30 \mathrm{~min}$ of treatment, the average number of filipin puncta per cell was slightly decreased, although it did not reach significance. However, the quantification revealed a shift towards cells with a lower number of puncta in the relative distribution (Fig. 6d, center). The average size of filipin puncta was reduced with a specific decrease in the number of large $\left(1.5-2.5 \mu \mathrm{m}^{2}\right)$ puncta (Fig. 6d, right). This partial rescue of cholesterol distribution was accompanied by a normalization of CI-M6PR distribution and an increase in NPC2/LAMP1 co-localization after PAO treatment (Supplementary Fig. 8b,c). Next, we overexpressed wild-type GFP-PIPKI $\gamma$ il (WT) or its kinase-dead K188A mutant in Arf6 KO MEFs (Fig. 7a). Quantification of filipin fluorescence in transfected cells after $48 \mathrm{~h}$ showed fewer filipin puncta of smaller size in WT-transfected cells (Fig. 7a). The kinase activity of PIPKI $\gamma$ il was thus required to rescue the cholesterol redistribution phenotype. To rule out that the changes in cholesterol distribution observed in the Arf6 $\mathrm{KO}$ were not due to the $\mathrm{PI}(4,5) \mathrm{P}_{2}$ levels increase we surprisingly observed after PAO treatment (Supplementary Fig. 8a), we used a third strategy to 
a

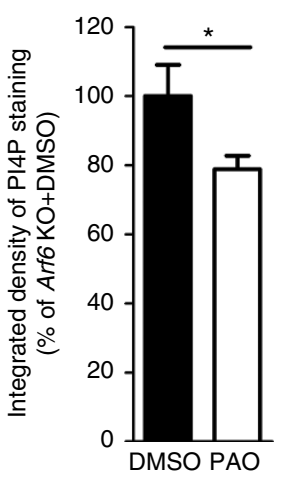

b

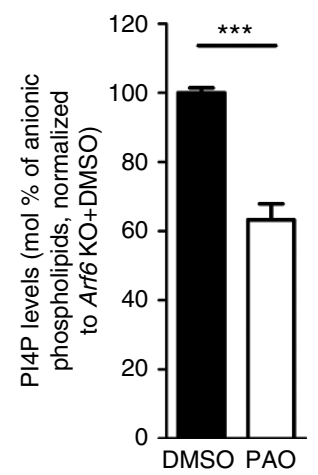

C

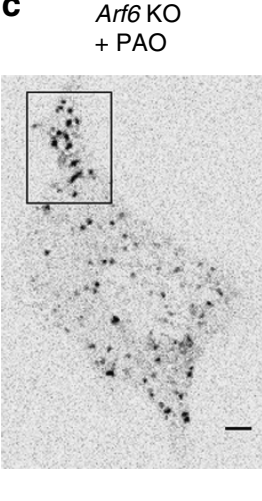

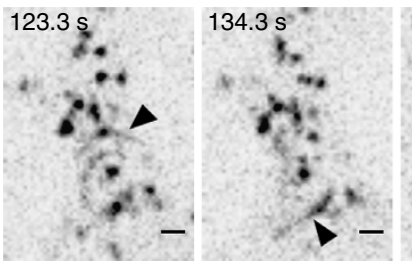
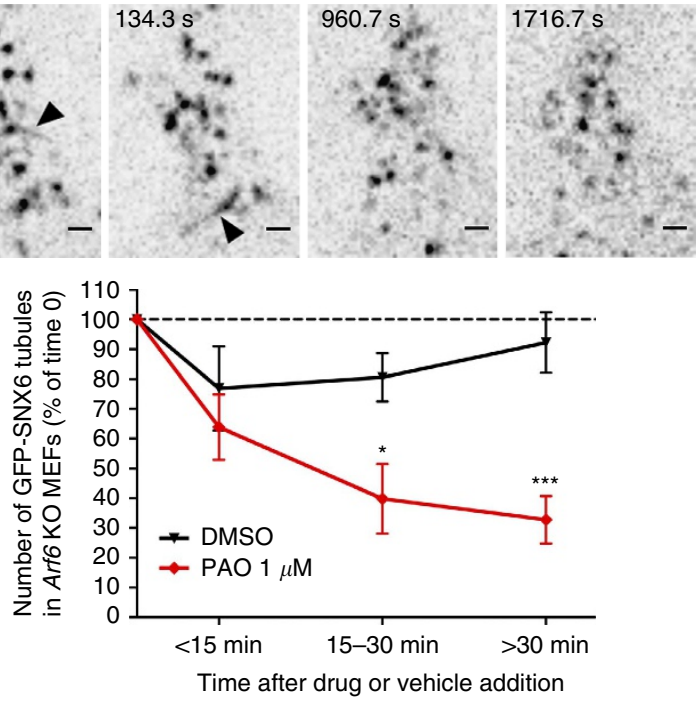

Arf6 KO PI4P
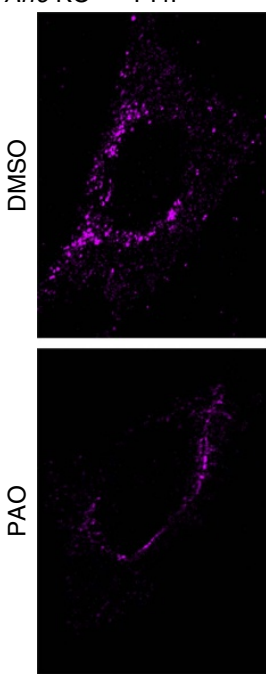

Filipin
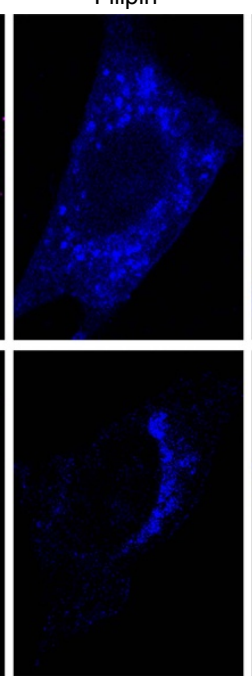
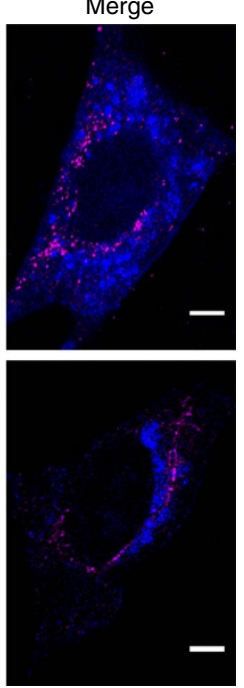
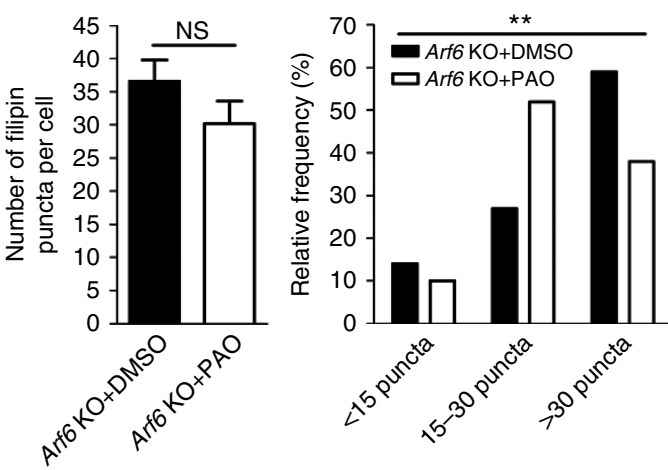

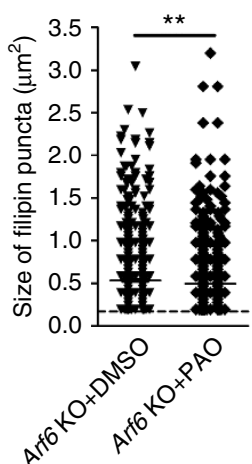

Figure 6 | Lowering PI4P levels with PAO rescues excessive retromer tubulation and cholesterol accumulation in LE/LYS in Arf6 KO cells.

(a) Normalized integrated densities of PI4P staining (see d) of Arf6 KO cells treated with DMSO (100 $\pm 9 \%, \pm$ indicates s.e.m., $n=22$ cells, 3 experiments) or PAO (79 $\pm 4 \%, n=29$ cells, three experiments). ${ }^{\star} P<0.05$ in $t$-test with Welch's correction. (b) Bar diagram showing PI4P levels in DMSO- and PAO-treated Arf6 KO cells. Measurements were made by anionic exchange HPLC with suppressed conductivity detection, expressed in molar percentage of measured lipids and normalized to DMSO-treated Arf6 KO levels. PI4P levels were decreased in PAO- $63 \pm 5 \%, \pm$ indicates s.e.m., $n=4)$ compared with DMSO-treated controls $(100 \pm 1 \%, n=4)$. ${ }^{\star \star \star} P<0.001$ in Student's $t$-test. (c) Kinetic following of GFP-SNX6 tubulation in Arf6 KO MEFs treated with DMSO or PAO by spinning-disk live imaging. Top panel, representative images of Arf6 KO cells after different times of PAO treatment. Arrows indicate tubules. Scale bar, 5 and $1 \mu \mathrm{m}$ (zoom-ins). Lower panel, quantification of the number of GFP-SNX 6 tubules after DMSO (black, $n=9$ cells, four experiments) or PAO treatment (red, $n=9$ cells, four experiments), normalized to the number of tubules before the treatment. ${ }^{\star} P<0.05$ and ${ }^{\star \star \star} P<0.001$ in two-way ANOVA with Bonferroni post-tests, respectively. (d) Left, representative immunostaining for PI4P (magenta) of Arf6 KO cells treated with DMSO or PAO and labelled with filipin (blue). Scale bar, $5 \mu \mathrm{m}$. Middle, quantification of the absolute number and relative distribution of filipin puncta in DMSO-(37 \pm 3 puncta/cell, \pm indicates s.e.m., $n=37$ cells, 4 experiments) or PAO-( $30 \pm 3$ puncta per cell, $n=29$ cells, four experiments) treated Arf6 KO cells. NS denotes $P>0.05$ in Student's $t$-test. ${ }^{\star \star} P<0.01$ in $\chi^{2}$-test. Right, size of filipin puncta in DMSO- $0.53 \pm 0.01 \mu \mathrm{m}^{2}, \pm$ indicates s.e.m., 1,352 puncta, 37 cells $)$ or PAO-(0.50 $\pm 0.01 \mu \mathrm{m}^{2}, 876$ puncta, 29 cells $)$ treated Arf6 KO cells. ${ }^{\star \star} P<0.01$ in Mann-Whitney test. NS, not significant.

selectively decrease PI4P levels in endosomes by exploiting the recently characterized endosomal PI4P-phosphatase Sac2 (refs 35,36) as a tool. We overexpressed wild-type GFP-Sac2 (WT) or its phosphatase-dead C458S mutant in Arf6 KO MEFs (Fig. 7b) and found that filipin puncta were fewer and of smaller size in WT GFP-Sac2 transfected cells (Fig. 7b). Cholesterol distribution rescue was thus dependent on the PI4P-phosphatase activity of Sac2. Altogether, these three approaches highlight the role of elevated PI4P levels in cholesterol accumulation in LE/LYS of Arf6 KO cells.

\section{Discussion}

Arf6 has been implicated in various aspects of cell physiology, primarily as a regulator of vesicular transport to and from the plasma membrane $e^{4,5}$. A key function of Arf6, however, appears to be the control of lipid signalling and, in particular, phosphoinositide signalling ${ }^{11}$. Parallel studies have also hinted that Arf6 may control cholesterol homeostasis, yet, through unknown mechanisms ${ }^{18-21}$. This study mechanistically links these disconnected bodies of literature, by identifying a phosphoinositide-based mechanism for the control of intracellular cholesterol distribution via the retromer pathway.

It is to our knowledge the first time that Arf6 is shown to regulate retromer function, although at least two previous studies presented circumstantial evidence consistent with this idea. First, even before retromer was identified, CI-M6PR was described as being present on Arf6-positive endosomes, but the functional relationship between these two proteins was not addressed ${ }^{3}$. Second, a more recent paper showed that EFA6A, a guanine 
a
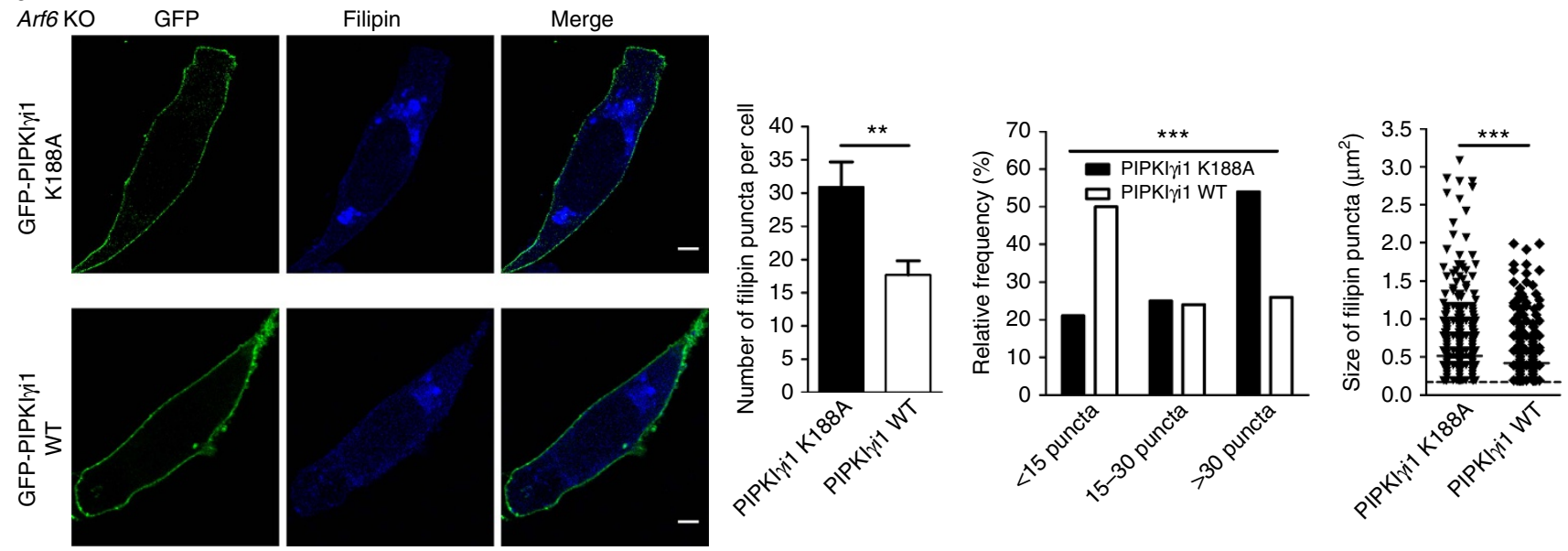

b
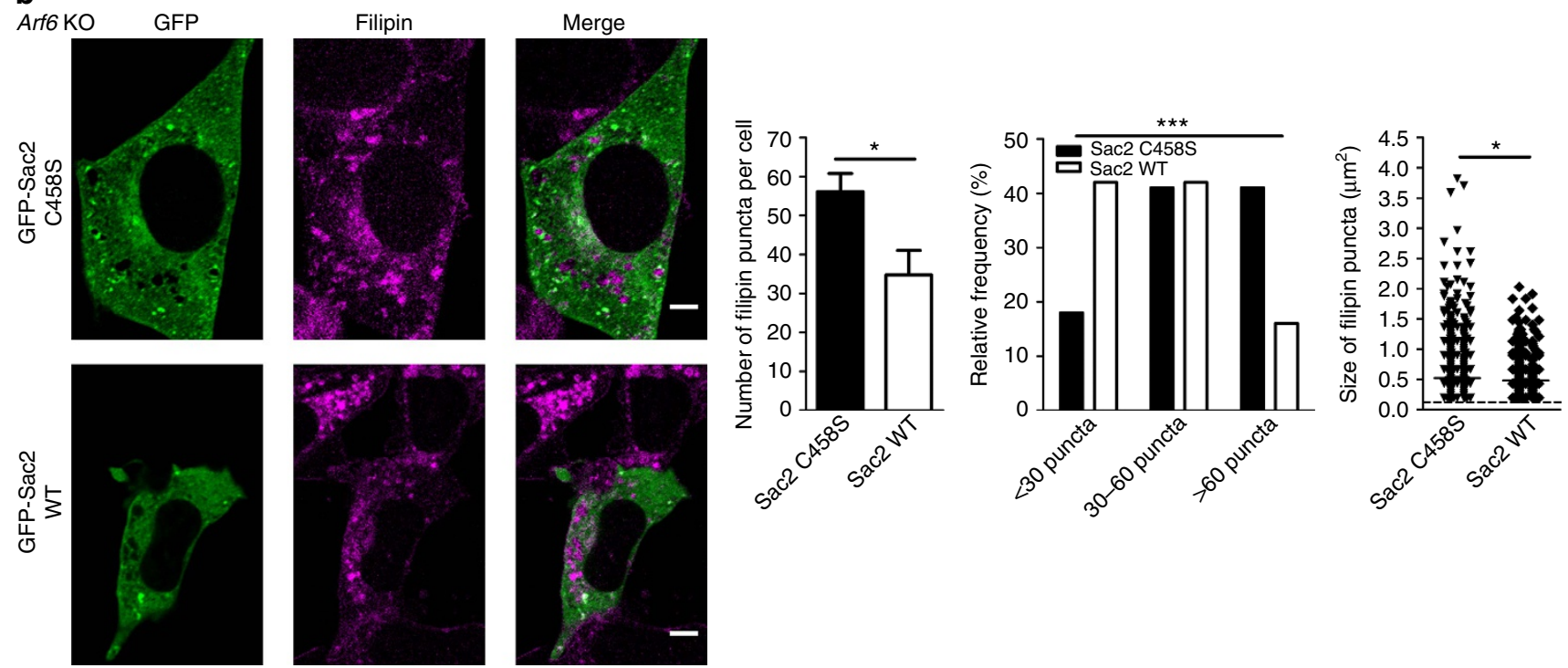

Figure 7 | Lowering PI4P levels through GFP-PIPKI $\gamma$ i1 or GFP-Sac2 overexpression rescues cholesterol redistribution in Arf6 KO cells. (a) Left, representative confocal images of Arf6 KO cells expressing the K188A mutant (top) or the wild-type (bottom) GFP-PIPKI $\gamma$ i1 (green) labelled with filipin (blue). Scale bar, $5 \mu \mathrm{m}$. Center, quantification of the absolute number and relative distribution of filipin puncta in Arf6 KO cells expressing K188A

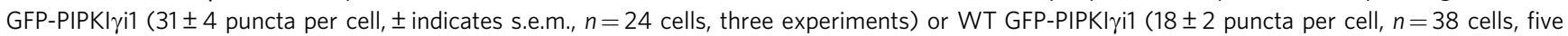
experiments). ${ }^{\star \star} P<0.01$ in $t$-test with Welch's correction. ${ }^{\star \star \star} P<0.001$ in $\chi^{2}$-test. Right, size of filipin puncta in Arf6 KO cells expressing K188A

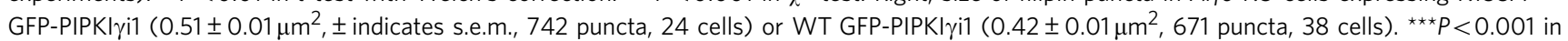
Mann-Whitney test. (b) Left, representative confocal images of Arf6 KO cells expressing the C458S mutant (top) or the wild-type (bottom) GFP-Sac2 (green) and labelled with filipin (magenta). Scale bar, $5 \mu \mathrm{m}$. Center, quantification of the absolute number and relative distribution of filipin puncta in Arf6 KO cells expressing C458S GFP-Sac2 ( $56 \pm 5$ puncta per cell, \pm indicates s.e.m., $n=27$ cells, three experiments) or WT GFP-Sac2 (35 \pm 6 puncta per cell, $n=12$ cells, three experiments). ${ }^{\star} P<0.05$ in Student's $t$-test. ${ }^{\star \star \star} P<0.001$ in $\chi^{2}$-test. Right, size of filipin puncta in Arf6 KO cells expressing C458S GFP-Sac2 $\left(0.52 \pm 0.01 \mu \mathrm{m}^{2}, \pm\right.$ indicates s.e.m., 1,517 puncta, 27 cells $)$ or WT GFP-Sac2 $\left(0.48 \pm 0.02 \mu \mathrm{m}^{2}, 417\right.$ puncta, 12 cells $) .{ }^{\star} P<0.05$ in Mann-Whitney test.

nucleotide exchange factor for Arf6, interacts with SNX1 and regulates neurite outgrowth through this interaction, although neither the role of retromer nor the link with phosphoinositide signalling and cholesterol homeostasis was addressed in that context $^{37}$. In this study, we propose a novel model whereby Arf6 controls the dynamics of retromer tubules in the endosome-toTGN pathway responsible for the retrograde transport of CIM6PR through negative regulation of an endosomal pool of PI4P (Fig. 8). Amongst the potential effectors of PI4P, specific members of the SNX family were considered excellent candidates based on their lipid-binding specificity. In fact, while SNX1, SNX2, SNX5 and SNX6 have all been shown to mediate the retromer-dependent traffic of CI-M6PR transport ${ }^{23,27-30}$ and bind to phosphoinositides via both their PX (phox homology) and BAR (Bin/ amphiphysin/ Rvs) domains, SNX6 appears to be the only member exhibiting a greater affinity for PI4P than for other phosphoinositides ${ }^{38}$, consistent with our findings showing defects in SNX6-coated tubule dynamics in Arf6 KO cells. Moreover, PI4P has been shown to compete with the p150(Glued) subunit of the dynein/dynactin complex for binding to SNX6 in the TGN ${ }^{38}$. Since impairing the retromer/ dynein motor coupling causes defects in retromer tubule fission ${ }^{39,40}$, an interesting possibility supported by our data is that the higher levels of PI4P found in the Arf6 KO cells may enhance the PI4P/SNX6 interaction on endosomal membranes at the expense of the SNX6/p150(Glued) interaction, resulting in 


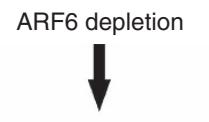

Increase in endosomal PI4P

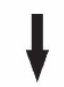

Aberrant retromer tubulation

$$
\checkmark
$$

Mistrafficking of Cl-M6PR

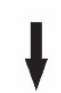

Mislocalization of NPC2
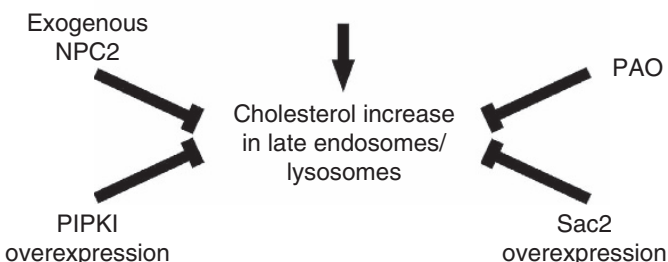

overexpression

Figure 8 | Model of Arf6 regulation of cholesterol homeostasis.

Arf6 controls an endosomal pool of PI4P and regulates retromer tubules dynamics in the endosome-to-TGN pathway, consequently impacting

$\mathrm{Cl}-\mathrm{M} 6 \mathrm{PR}$ and NPC2 localization (see also text). PAO: PI4K inhibitor.

retromer fission defects. Whether the lack of Arf6 and its $\mathrm{NH}_{2}$ terminal amphipathic helix directly alters membrane tubule dynamics as described for other Arf family members ${ }^{41,42}$, and contributes to retromer tubules defects, remains to be investigated. Similarly, a perturbation of the $\mathrm{PI} 4 \mathrm{P} / \mathrm{PI}(4,5) \mathrm{P}_{2}$ ratio on endosomal membranes may alter the fission efficiency of retromer tubules as we have previously reported for endocytic structures ${ }^{43}$.

Our study also further highlights the tight crosstalk between small GTPases and phosphoinositide metabolism ${ }^{32,44}$. In the context of retromer regulation, Rab5 has been shown to control class III PI3K (ref. 45), whose product PI3P mediates the recruitment of key components of the retromer assemblies, SNX1 and SNX2 (refs 27-29,46). In contrast, Rab7 directly interacts with the VPS core complex to facilitate the recruitment of retromer to endosomal membranes ${ }^{46,47}$. In this study, we propose a novel mechanism for GTPase-mediated control of retromer, involving the negative regulation of endosomal PI4P levels. As mentioned earlier, Arf6 controls PIPKIs, suggesting that PI $(4,5) \mathrm{P}_{2}$ generation may be key to its biological function. However, the HPLC profile suggests that it is rather the accumulation of PI4P on endosomes than a reduction in $\mathrm{PI}(4,5) \mathrm{P}_{2}$ which accounts for the retromer phenotype. This is further supported by our rescue experiments showing that decreasing PI4P levels, rather than enhancing $\mathrm{PI}(4,5) \mathrm{P}_{2}$ levels, corrects the retromer phenotype (Figs 6 and 7). In addition, because PIPKI $\gamma$ overexpression rescues the cholesterol redistribution phenotype, we speculate that dysregulation of a PIPKI isoform on endosomal membranes is the basis of PI4P accumulation on these compartments. However, we cannot rule out contributions of class II PI3Ks, PI4Ks or PI4P phosphatases to this phenotype, particularly in light of the presence of some of their isoforms in the endosomal compartments, including the Sac2 inositol 4-phosphatase ${ }^{34-36,48-55}$.

While our study further establishes a link between Arf6 and NPC disease ${ }^{19}$, it also reports a role of retromer in endolysosomal cholesterol homeostasis. As retromer has been linked to several

neurodegenerative diseases ${ }^{56}$ and cholesterol accumulation in LE/LYS can lead to neurodegeneration ${ }^{57}$, this raises the question of the importance of the link between retromer and cholesterol in neurodegeneration. It is particularly intriguing that both decreased retromer levels ${ }^{58}$ and increased cholesterol levels ${ }^{59,60}$ have been linked to late-onset Alzheimer's disease. Further studies should give us insights into the role of the novel Arf6/retromer/cholesterol connection in the aetiology of neurodegenerative diseases.

\section{Methods}

Reagents. DMSO, tamoxifen, filipin, phenylarsine oxide (PAO) and bovine serum albumin (BSA) were purchased from Sigma-Aldrich. Saponin was from Acros.

Antibodies and peptides. The antibody against Arf6 (ref. 61) was a kind gift from Julie Donaldson (NIH, Bethesda, MD; 1/1000 for western blots). The other antibodies were obtained from the following sources: rabbit antibodies to LAMP1 (ab24170, Abcam, 1/500 in immunofluorescence (IF), 1/500 for western blots), Rab7 (9367, Cell Signalling Technology, 1/100 in IF), EEA1 (3288, Cell Signalling Technology, 1/400 in IF), NPC1 (ab36983, Abcam, 1/500 for western blots), NPC2 (sc-33776, Santa Cruz, 1/50 in IF and H00010577-D01P, Abnova, 1/100 for western blots), M6PR (PA3-850, Thermo, 1/100 in IF), Golgin97 (13192, Cell Signalling Technology, 1/100 in IF); goat polyclonal antibodies to SNX1 (ab99286, Abcam, 1/50 in IF), SNX6 (sc-8679, Santa Cruz, 1/50 in IF) and Vps35 (ab10099, Abcam, 1/150 in IF); rat monoclonal antibodies (mABs) to LAMP1 (sc-19992, Santa Cruz, 1/50 in IF) and HA (3F10, Roche, 1/200 in IF); mouse monoclonal antibodies to tubulin (T5168, Sigma, 1/1000 for western blots), NPC2 (sc-166449, Santa Cruz, $1 / 50$ in IF), Vps35 (ab57632, Abcam, 1/300 in IF, 1/1,000 for western blots) and PI4P (Z-P004, Echelon, 1/100 in IF). Purified NPC2-Alexa488 (ref. 62) was a kind gift from Ling Huang and Peter Lobel (Rutgers University, Piscataway, NJ). Peroxidase-conjugated secondary antibodies were from Biorad and were used at a dilution 1/3,000. Alexa-conjugated fluorescent secondary antibodies were from Life Technologies (1/400 for Alexa 488 and Alexa 555; 1/300 for Alexa 647).

Plasmids and RNA interference. NPC1-GFP was kindly provided by Kara Primmer and Kevin Vaughan (University of Notre Dame, Notre Dame, IN) ${ }^{63}$. GFP-SNX6 was kindly provided by Peter Cullen (University of Bristol, UK) ${ }^{30}$. ARF6-HA ${ }^{3}$ was kindly provided by Julie Donaldson (NIH, Bethesda, MD) and CyPET-ARF6 (ref. 31) was a kind gift from Martin Schwartz (Addgene plasmid \# 18840). GFP-Sac2 and its phosphatase-dead mutant GFP-Sac2 C458S were kind gifts from Yuxin Mao (Cornell University, Ithaca, NY) ${ }^{35}$. GFP-PIPKI $\gamma$ i1 and its kinase-dead mutant GFP-PIPKI $\gamma$ i1-K188A were previously published ${ }^{64}$. All DNA sequences were checked by sequencing. VPS35 knockdown (KD) was performed with a 1:1 mix of Qiagen Flexitube siRNA Human VPS35_5 (SI04268131) and Human Vps35_6 (SI04279296). SNX1 and SNX2 double knockdowns were performed with a 1:1 mix of ON-TARGET plus Human SNX1 (6642) siRNA-SMART pool and ON-TARGET plus Human SNX2 (6643) siRNA-SMART pool (GE Dharmacon). SNX5 and SNX6 double knockdowns were performed with a 1:1 mix of ON-TARGET plus Human SNX5 (27131) siRNA-SMART pool and ON-TARGET plus Human SNX6 (58533) siRNA-SMART pool (GE Dharmacon).

Generation of Arf6 ${ }^{\text {Flox/Flox }}$ mice. To generate Arf6 conditional KO mice, the entire coding sequence $(\sim 4,000 \mathrm{bp})$ was flanked with loxP sites, with one (loxP) sub-cloned into a targeting vector upstream of the Arf6 coding region at the SspI site and a FRT-NEO-FRT-loxP cassette inserted downstream of the Arf6 sequence at the XhoI site. The targeting vector was electroporated into ES cells and successfully targeted ES cells were microinjected into blastocysts, which were then transferred into foster mothers for the generation of chimeric mice. Resultant

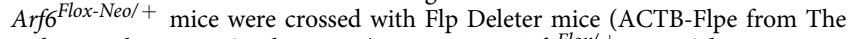
Jackson Laboratory, Stock 005703) to generate Arf6 $6^{\text {Flox } /+}$ mice. These mice were then crossed with the inducible Cre-ER mice (Rosa B6 ${ }^{\mathrm{Cre}-\mathrm{ER} / \mathrm{Cre}-\mathrm{ER}}$ from the Jackson Laboratory, Stock 008463), to generate Arf6 Flox/+ ;Cre-ER mice, which were then interbred to generate Arf6 ${ }^{\text {Flox/Flox }}$;Cre-ER mice. Animals were used in full compliance with National Institutes of Health/ Institutional Animal Care and Use Committee guidelines. Specifically, adult mice were sacrificed via inhalation of carbon dioxide, followed by cervical dislocation, while embryos derived from the killed mothers were decapitated after inducing hypothermia on ice. The animal protocols were approved by the Committee on the Ethics of Animal Experiments of Columbia University. Arf6 Flox/ $^{+}$mice were made available to The Jackson Laboratory.

Cell cultures and transfection. Immortalized MEFs and HeLa cells were maintained at $37^{\circ} \mathrm{C}$ in a humidified $5 \% \mathrm{CO}_{2}$ atmosphere in DMEM with Glutamax supplemented with $10 \%$ fetal bovine serum and $1 \%$ penicillin/streptomycin (all from Life technologies). Cells were negative for mycoplasma contamination. Primary MEFs were generated from embryonic day 13.5 Arf6 ${ }^{\text {Flox/Flox; }}$ Cre-ER mice. 
Torsos from Arf6 Flox/Flox;Cre-ER embryos were minced and trypsinized for 15 min and homogenized by pipetting. MEFs were immortalized by multiple passaging and were used for experiments after 25 passages. Arf6 ${ }^{\text {Flox/Flox; }}$ Cre-ER MEFs contain a Cre-oestrogen receptor (Cre-ER) fusion protein, rendering Cre recombinase

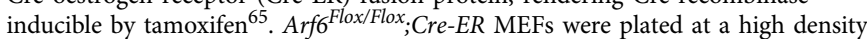
and treated the next day with DMSO or $10 \mu \mathrm{M}$ tamoxifen. After $72 \mathrm{~h}$, cells were split in fresh media and left in culture for another $72 \mathrm{~h}$. Arf6 deletion was complete by then. All experiments were performed between 3 and 7 days after complete Arf6 deletion. MEFs were transiently transfected using Lipofectamine LTX (Life Technologies) and protein expression was assessed after 24 or $48 \mathrm{~h}$. HeLa cells were transfected using Lipofectamine 2000 (Life Technologies). Protein expression was assessed after 24 or $48 \mathrm{~h}$ and knockdowns were assessed at $72 \mathrm{~h}$.

Anionic phospholipid analysis. Phosphoinositides and other anionic phospholipids were measured as deacylated lipids using anionic exchange HPLC with suppressed conductivity detection ${ }^{66,67}$. Samples were prepared from cells grown to half-confluency in 100 -mm dishes, washed with ice-cold HBSS and processed for phosphoinositide enrichment, followed by deacylation ${ }^{66,67}$.

Free cholesterol analysis. Free cholesterol levels (and other lipids) were measured by separating complex lipid mixtures by reverse phase HPLC, followed by detection using multiple reactions monitoring ${ }^{68}$. Samples were prepared from cells grown to half-confluency in 60 -mm dishes, washed with ice-cold HBSS and processed for lipid extraction using a modified Bligh and Dyer protocol ${ }^{68}$. Free cholesterol levels were quantified by referencing a D7-cholesterol (Avanti Polar Lipids) internal standard that was spiked into each sample before the analysis.

Immunofluorescence and filipin staining. For NPC2 uptake experiments, MEFs were exposed to fresh medium containing PBS (Boston BioProducts) or purified NPC2-Alexa488 (ref. 62) $60 \mathrm{nM}$ for $24 \mathrm{~h}$, rinsed twice with HBSS (Life Technologies) and fixed. For PAO experiments, MEFs were exposed to fresh medium containing DMSO or PAO $1 \mu \mathrm{M}$ for $30 \mathrm{~min}$ at $37^{\circ} \mathrm{C}$, rinsed twice with HBSS and fixed. For immunofluorescence experiments, cells grown on glass coverslips were washed once with HBSS and fixed with $4 \%$ paraformaldehyde (Electron Microscopy Sciences) for $20 \mathrm{~min}$ at room temperature or at $37^{\circ} \mathrm{C}$ (when preservation of the tubular structures was crucial). Cells were then washed twice in HBSS and incubated with $\mathrm{NH} 4 \mathrm{Cl}(50 \mathrm{mM}$ in PBS) for $10 \mathrm{~min}$. For NPC2 staining, cells were fixed for $1 \mathrm{~h}$ in Bouin's solution (Sigma-Aldrich). Cells were then washed twice in PBS and permeabilized with solution A (saponin 0.05\%, BSA 5\% in PBS) for at least $45 \mathrm{~min}$ at $37^{\circ} \mathrm{C}$. They were then incubated with primary antibodies diluted in solution A for $1 \mathrm{~h}$ at room temperature, washed three times in solution $\mathrm{A}$, incubated with fluorescent secondary antibodies diluted in solution $\mathrm{A}$ for $1 \mathrm{~h}$ at room temperature and washed again three times in solution A. An extra step was performed for filipin staining. Cells were washed once in PBS, incubated in filipin $\left(0.5 \mathrm{mg} \mathrm{ml}^{-1}\right.$ in PBS) for $1 \mathrm{~h}$ at room temperature and washed twice with PBS. Cells were finally washed once with PBS and coverslips were mounted in Vectashield mounting medium (Vectorlabs).

Confocal microscopy. Z-stacks were acquired by confocal laser scanning microscopy (Zeiss LSM 700). Fluorescence was collected with a $\times 63$ plan apochromat immersion oil objective (numerical aperture (NA) 1.4). Extraction of single $\mathrm{z}$-frame and maximum intensity projections were performed with the ImageJ software. Integrated densities, that is, cell area $\times[$ (mean cell intensities summed on the whole $\mathrm{z}$-stack)-(mean background intensities summed on the whole $\mathrm{z}$-stack)], were measured with Image J software. Z-stack co-localization percentage, that is, $\times 100$ (Pearson's coefficient) $^{2}$, were obtained with the JACoP plugin of Image ${ }^{69}$. The number and size of puncta were quantified with ICY software ${ }^{70}$. We checked that the cells we were comparing for fluorescence intensity, size and number of organelles or co-localization had comparable areas $(P>0.05$ in Student's $t$-test). Each experiment was independently repeated at least three times, resulting in the analysis of 22 to 42 cells for each condition.

3D Structured illumination microscopy. Cells were grown on high performance \#1.5 coverslips (Zeiss). Immunofluorescence was performed as described for confocal microscopy but secondary antibodies were used twice as concentrated $(1 / 200)$ and coverslips were mounted in ProLong Gold (Life technologies). Z-stacks were acquired on a Nikon N-SIM microscope equipped with an iXon Ultra 897 camera (Andor) and a $\times 100$ plan apochromat immersion oil objective (NA 1.49). Image reconstruction and analysis were performed with the NIS-Elements software.

Spinning-disk confocal live imaging. Cells were grown in $35-\mathrm{mm}$ glass-bottom dishes (MatTek) and imaged $48 \mathrm{~h}$ after transfection. Before imaging, medium was replaced to get rid of any debris and to ensure accuracy of volume for the PAO experiments. For kinetic PAO experiments, Arf6 KO cells expressing GFP-SNX6 were imaged for $2 \mathrm{~min}$ and their localization recorded. At time 0 , DMSO or PAO
( $1 \mu \mathrm{M}$ final) diluted in medium was added to the cells and three movies of $2 \mathrm{~min}$ were recorded (before $15 \mathrm{~min}$, between 15 and $30 \mathrm{~min}$ and between 30 and $45 \mathrm{~min}$ ). Only cells displaying at least two tubules at time 0 were kept for analysis. Live imaging was carried out using a Nikon Ti Eclipse microscope equipped with a Yokogawa CSU-X1 spinning-disk, a motorized stage and a Photometrics Evolve 512 camera (Roper Scientific) controlled with the NIS-Elements software. Movies were acquired at $37^{\circ} \mathrm{C}$ and $5 \% \mathrm{CO}_{2}$ with a Tokai Hit stage-top incubator and objective heater. Fluorescence was collected with a $60 \times$ plan apochromat immersion oil objective (NA 1.49). Images were collected at $1 \times 1$ binning with an exposure time of $100 \mathrm{~ms}$. The number, maximum length and persistence of GFP-SNX6 tubules were analysed with ImageJ software.

Protein biochemistry and immunoblotting. Cells were washed with ice-cold HBSS, scraped and centrifuged for $10 \mathrm{~min}$ at $16,000 \mathrm{~g}$ at $4^{\circ} \mathrm{C}$. Pellets were resuspended in RIPA buffer (Pierce) complemented with cOmplete protease inhibitor cocktail (Roche) and proteins were extracted on a wheel at $4{ }^{\circ} \mathrm{C}$ for at least $30 \mathrm{~min}$. Samples were then centrifuged for $15 \mathrm{~min}$ at $16,000 \mathrm{~g}$ and proteins in the supernatant were processed for protein dosage (BCA, Pierce). SDS-PAGE was performed on samples ( $15 \mu \mathrm{g}$ total proteins) loaded in NuPAGE $4-12 \%$ Bis-Tris gels (Life technologies). For quantification, wet transfer was performed at $40 \mathrm{~V}$ for $135 \mathrm{~min}$ at $4{ }^{\circ} \mathrm{C}$ (Bio-Rad). For Arf6 deletion confirmation, transfer was performed with iBlot (Life Technologies). Primary and secondary antibodies were incubated overnight at $4{ }^{\circ} \mathrm{C}$ and $45 \mathrm{~min}$ at room temperature, respectively. Revelation was performed with Immobilon Western Chemiluminescent HRP Substrate (EMD Millipore) and the chemiluminescent signal was imaged with ImageQuant LAS4000 mini (GE Healthcare). Quantification was performed with ImageJ. Uncropped blots are shown in Supplementary Fig. 9.

Statistical analysis. Statistical calculations were performed using GraphPad Prism software (version 5.02). All the data are given as mean \pm s.e.m. In most cases, when comparing two samples, two-tailed Student's $t$-test was performed. If a clear hypothesis was postulated that made it valid, then we used a one-tailed $t$-test. When variances were not comparable, Welch's correction was applied. When the distribution could not be assumed to be Gaussian (for compartments size), we used a non parametric Mann-Whitney test. When more samples were compared and Bartlett's test showed that variances could be compared, we used one-way ANOVA or two-way ANOVA with Bonferroni post-tests. If variances could not be compared $(P$ value in Bartlett's test $<0.05)$, then we used $t$-tests. Chi-square test was used to compare distributions. Outliers, defined as values that were superior to (mean $+3 \times$ s.d.) or inferior to (mean $-3 \times$ s.d.) were excluded.

Data availability. The authors declare that all the data supporting the findings of this study are available within the article and its Supplementary Information files or are available from the corresponding authors on request. Arf $6^{\mathrm{Flox} /+}$ mice generated in this study have been made available to the Jackson Laboratory.

\section{References}

1. Gillingham, A. K. \& Munro, S. The small G proteins of the Arf family and their regulators. Annu. Rev. Cell Dev. Biol. 23, 579-611 (2007).

2. D'Souza-Schorey, C., Li, G., Colombo, M. I. \& Stahl, P. D. A regulatory role for ARF6 in receptor-mediated endocytosis. Science 267, 1175-1178 (1995).

3. Peters, P. J. et al. Overexpression of wild-type and mutant ARF1 and ARF6: distinct perturbations of nonoverlapping membrane compartments. J. Cell Biol. 128, 1003-1017 (1995).

4. Donaldson, J. G. Multiple roles for Arf6: sorting, structuring, and signalling at the plasma membrane. J. Biol. Chem. 278, 41573-41576 (2003).

5. D'Souza-Schorey, C. \& Chavrier, P. ARF proteins: roles in membrane traffic and beyond. Nat. Rev. Mol. Cell Biol. 7, 347-358 (2006).

6. Ghossoub, R. et al. Syntenin-ALIX exosome biogenesis and budding into multivesicular bodies are controlled by ARF6 and PLD2. Nat. Commun. 5, 3477 (2014).

7. Jaworski, J. ARF6 in the nervous system. Eur. J. Cell Biol. 86, 513-524 (2007).

8. Suzuki, T. et al. Crucial role of the small GTPase ARF6 in hepatic cord formation during liver development. Mol. Cell Biol. 26, 6149-6156 (2006).

9. Akiyama, M. et al. Trans-regulation of oligodendrocyte myelination by neurons through small GTPase Arf6-regulated secretion of fibroblast growth factor-2. Nat. Commun. 5, 4744 (2014).

10. Honda, A. et al. Phosphatidylinositol 4-phosphate 5-kinase alpha is a downstream effector of the small G protein ARF6 in membrane ruffle formation. Cell 99, 521-532 (1999).

11. Funakoshi, Y., Hasegawa, H. \& Kanaho, Y. Activation mechanisms of PIP5K isozymes by the small GTPase ARF6. Adv. Enzyme Regul. 50, 72-80 (2010).

12. Massenburg, D. et al. Activation of rat brain phospholipase D by ADP-ribosylation factors 1,5, and 6: separation of ADP-ribosylation factor-dependent and oleate-dependent enzymes. Proc. Natl Acad. Sci. USA 91, 11718-11722 (1994).

13. Roth, M. G. Molecular mechanisms of PLD function in membrane traffic. Traffic 9, 1233-1239 (2008). 
14. Brown, F. D., Rozelle, A. L., Yin, H. L., Balla, T. \& Donaldson, J. G. Phosphatidylinositol 4,5-bisphosphate and Arf6-regulated membrane traffic. J. Cell Biol. 154, 1007-1017 (2001).

15. Brown, M. S. \& Goldstein, J. L. A proteolytic pathway that controls the cholesterol content of membranes, cells, and blood. Proc. Natl Acad. Sci. USA 96, 11041-11048 (1999).

16. Rosenbaum, A. I. \& Maxfield, F. R. Niemann-Pick type C disease: molecular mechanisms and potential therapeutic approaches. J. Neurochem. 116, 789-795 (2011).

17. Vance, J. E. \& Karten, B. Niemann-Pick C disease and mobilization of lysosomal cholesterol by cyclodextrin. J. Lipid Res. 55, 1609-1621 (2014).

18. Naslavsky, N., Weigert, R. \& Donaldson, J. G. Characterization of a nonclathrin endocytic pathway: membrane cargo and lipid requirements. Mol. Biol. Cell 15, 3542-3552 (2004).

19. Schweitzer, J. K., Pietrini, S. D. \& D’Souza-Schorey, C. ARF6-mediated endosome recycling reverses lipid accumulation defects in Niemann-Pick Type C disease. PloS ONE 4, e5193 (2009).

20. Marquer, C. et al. Increasing membrane cholesterol of neurons in culture recapitulates Alzheimer's disease early phenotypes. Mol. Neurodegener. 9, 60 (2014).

21. Chu, B. B. et al. Cholesterol transport through lysosome-peroxisome membrane contacts. Cell 161, 291-306 (2015)

22. Willenborg, M. et al. Mannose 6-phosphate receptors, Niemann-Pick C2 protein, and lysosomal cholesterol accumulation. J. Lipid Res. 46, 2559-2569 (2005).

23. Seaman, M. N. Cargo-selective endosomal sorting for retrieval to the Golgi requires retromer. J. Cell Biol. 165, 111-122 (2004).

24. Arighi, C. N., Hartnell, L. M., Aguilar, R. C., Haft, C. R. \& Bonifacino, J. S. Role of the mammalian retromer in sorting of the cation-independent mannose 6-phosphate receptor. J. Cell Biol. 165, 123-133 (2004).

25. Geuze, H. J. et al. Sorting of mannose 6-phosphate receptors and lysosomal membrane proteins in endocytic vesicles. J. Cell Biol. 107, 2491-2501 (1988)

26. Cullen, P. J. \& Korswagen, H. C. Sorting nexins provide diversity for retromer-dependent trafficking events. Nat. Cell Biol. 14, 29-37 (2012).

27. Carlton, J. et al. Sorting nexin-1 mediates tubular endosome-to-TGN transport through coincidence sensing of high-curvature membranes and 3-phosphoinositides. Curr. Biol. 14, 1791-1800 (2004).

28. Carlton, J. G. et al. Sorting nexin-2 is associated with tubular elements of the early endosome, but is not essential for retromer-mediated endosome-to-TGN transport. J. Cell Sci. 118, 4527-4539 (2005).

29. Rojas, R., Kametaka, S., Haft, C. R. \& Bonifacino, J. S. Interchangeable but essential functions of SNX1 and SNX2 in the association of retromer with endosomes and the trafficking of mannose 6-phosphate receptors. Mol. Cell Biol. 27, 1112-1124 (2007).

30. Wassmer, T. et al. A loss-of-function screen reveals SNX5 and SNX6 as potential components of the mammalian retromer. J. Cell Sci. 120, 45-54 (2007).

31. Hall, B. et al. A fluorescence resonance energy transfer activation sensor for Arf6. Anal. Biochem. 374, 243-249 (2008).

32. Di Paolo, G. \& De Camilli, P. Phosphoinositides in cell regulation and membrane dynamics. Nature 443, 651-657 (2006).

33. Hammond, G. R., Machner, M. P. \& Balla, T. A novel probe for phosphatidylinositol 4-phosphate reveals multiple pools beyond the Golgi. J. Cell Biol. 205, 113-126 (2014).

34. Balla, A., Tuymetova, G., Barshishat, M., Geiszt, M. \& Balla, T. Characterization of type II phosphatidylinositol 4-kinase isoforms reveals association of the enzymes with endosomal vesicular compartments. J. Biol. Chem. 277, 20041-20050 (2002).

35. Hsu, F., Hu, F. \& Mao, Y. Spatiotemporal control of phosphatidylinositol 4-phosphate by Sac2 regulates endocytic recycling. J. Cell Biol. 209, 97-110 (2015).

36. Nakatsu, F. et al. Sac2/INPP5F is an inositol 4-phosphatase that functions in the endocytic pathway. J. Cell Biol. 209, 85-95 (2015).

37. Fukaya, M., Fukushima, D., Hara, Y. \& Sakagami, H. EFA6A, a guanine nucleotide exchange factor for Arf6, interacts with sorting nexin-1 and regulates neurite outgrowth. J. Neurochem. 129, 21-36 (2014).

38. Niu, Y. et al. PtdIns(4)P regulates retromer-motor interaction to facilitate dynein-cargo dissociation at the trans-Golgi network. Nat. Cell Biol. 15, 417-429 (2013)

39. Wassmer, T. et al. The retromer coat complex coordinates endosomal sorting and dynein-mediated transport, with carrier recognition by the trans-Golgi network. Dev. Cell 17, 110-122 (2009).

40. Hong, Z. et al. The retromer component SNX6 interacts with dynactin p150(Glued) and mediates endosome-to-TGN transport. Cell. Res. 19, 1334-1349 (2009).

41. Lee, M. C. et al. Sarlp N-terminal helix initiates membrane curvature and completes the fission of a COPII vesicle. Cell 122, 605-617 (2005).

42. Krauss, M. et al. Arf1-GTP-induced tubule formation suggests a function of Arf family proteins in curvature acquisition at sites of vesicle budding. J. Biol. Chem. 283, 27717-27723 (2008).
43. Chang-Ileto, B. et al. Synaptojanin 1-mediated PI(4,5)P2 hydrolysis is modulated by membrane curvature and facilitates membrane fission. Dev. Cell 20, 206-218 (2011).

44. Jean, S. \& Kiger, A. A. Coordination between RAB GTPase and phosphoinositide regulation and functions. Nat. Rev. Mol. Cell Biol. 13, 463-470 (2012).

45. Christoforidis, S. et al. Phosphatidylinositol-3-OH kinases are Rab5 effectors. Nat. Cell Biol. 1, 249-252 (1999).

46. Rojas, R. et al. Regulation of retromer recruitment to endosomes by sequential action of Rab5 and Rab7. J. Cell Biol. 183, 513-526 (2008).

47. Seaman, M. N., Harbour, M. E., Tattersall, D., Read, E. \& Bright, N. Membrane recruitment of the cargo-selective retromer subcomplex is catalysed by the small GTPase Rab7 and inhibited by the Rab-GAP TBC1D5. J. Cell Sci. 122, 2371-2382 (2009).

48. Salazar, G. et al. Phosphatidylinositol-4-kinase type II alpha is a component of adaptor protein-3-derived vesicles. Mol. Biol. Cell 16, 3692-3704 (2005).

49. Minogue, S. et al. Phosphatidylinositol 4-kinase is required for endosomal trafficking and degradation of the EGF receptor. J. Cell Sci. 119, 571-581 (2006).

50. Burgess, J. et al. Type II phosphatidylinositol 4-kinase regulates trafficking of secretory granule proteins in Drosophila. Development 139, 3040-3050 (2012).

51. Jovic, M. et al. Two phosphatidylinositol 4-kinases control lysosomal delivery of the Gaucher disease enzyme, beta-glucocerebrosidase. Mol. Biol. Cell 23, 1533-1545 (2012).

52. Falasca, M. \& Maffucci, T. Regulation and cellular functions of class II phosphoinositide 3-kinases. Biochem. J. 443, 587-601 (2012).

53. Wieffer, M. et al. PI4K2beta/AP-1-based TGN-endosomal sorting regulates Wnt signalling. Curr. Biol. 23, 2185-2190 (2013).

54. Jovic, M. et al. Endosomal sorting of VAMP3 is regulated by PI4K2A. J. Cell Sci. 127, 3745-3756 (2014)

55. Jean, S. \& Kiger, A. A. Classes of phosphoinositide 3-kinases at a glance. J. Cell Sci. 127, 923-928 (2014).

56. Small, S. A. \& Petsko, G. A. Retromer in Alzheimer disease, Parkinson disease and other neurological disorders. Nat. Rev. Neurosci. 16, 126-132 (2015).

57. Aqul, A. et al. Unesterified cholesterol accumulation in late endosomes/lysosomes causes neurodegeneration and is prevented by driving cholesterol export from this compartment. J. Neurosci. 31, 9404-9413 (2011).

58. Small, S. A. et al. Model-guided microarray implicates the retromer complex in Alzheimer's disease. Ann. Neurol. 58, 909-919 (2005).

59. Cutler, R. G. et al. Involvement of oxidative stress-induced abnormalities in ceramide and cholesterol metabolism in brain aging and Alzheimer's disease. Proc. Natl Acad. Sci. USA 101, 2070-2075 (2004).

60. Lazar, A. N. et al. Time-of-flight secondary ion mass spectrometry (TOF-SIMS) imaging reveals cholesterol overload in the cerebral cortex of Alzheimer disease patients. Acta. Neuropathol. 125, 133-144 (2013)

61. Song, J., Khachikian, Z., Radhakrishna, H. \& Donaldson, J. G. Localization of endogenous ARF6 to sites of cortical actin rearrangement and involvement of ARF6 in cell spreading. J. Cell Sci. 111, 2257-2267 (1998).

62. Liou, H. L. et al. NPC2, the protein deficient in Niemann-Pick C2 disease, consists of multiple glycoforms that bind a variety of sterols. J. Biol. Chem. 281, 36710-36723 (2006).

63. Yi, J. Y. et al. High-resolution imaging reveals indirect coordination of opposite motors and a role for LIS1 in high-load axonal transport. J. Cell Biol. 195, 193-201 (2011).

64. Di Paolo, G. et al. Recruitment and regulation of phosphatidylinositol phosphate kinase type 1 gamma by the FERM domain of talin. Nature 420, 85-89 (2002).

65. Hayashi, S. \& McMahon, A. P. Efficient recombination in diverse tissues by a tamoxifen-inducible form of Cre: a tool for temporally regulated gene activation/inactivation in the mouse. Dev. Biol. 244, 305-318 (2002).

66. Nasuhoglu, C. et al. Nonradioactive analysis of phosphatidylinositides and other anionic phospholipids by anion-exchange high-performance liquid chromatography with suppressed conductivity detection. Anal. Biochem. 301, 243-254 (2002)

67. Berman, D. E. et al. Oligomeric amyloid-beta peptide disrupts phosphatidylinositol-4,5-bisphosphate metabolism. Nat. Neurosci. 11, 547-554 (2008).

68. Chan, R. B. et al. Comparative lipidomic analysis of mouse and human brain with Alzheimer disease. J. Biol. Chem. 287, 2678-2688 (2012).

69. Bolte, S. \& Cordelieres, F. P. A guided tour into subcellular colocalization analysis in light microscopy. J. Microsc. 224, 213-232 (2006).

70. de Chaumont, F. et al. Icy: an open bioimage informatics platform for extended reproducible research. Nat. Methods. 9, 690-696 (2012).

\section{Acknowledgements}

We thank Julie Donaldson (NIH, Bethesda, MD) for the kind gift of Arf6 antibody and ARF6-HA plasmid as well as Ling Huang and Peter Lobel (Rutgers University, Piscataway, NJ) for kindly providing us with purified NPC2-Alexa488. We also would 
like to thank Kara Primmer and Kevin Vaughan (University of Notre Dame, Notre Dame, IN), Peter Cullen (University of Bristol, UK), Martin Schwartz (Yale School of Medicine, New Haven, CT) and Yuxin Mao (Cornell University, Ithaca, NY) for their kind gift of NPC1-GFP, CyPET-ARF6, GFP-SNX6 and GFP-Sac2 plasmids, respectively. We are especially grateful to Dr. Scott Small for fruitful discussions. We would like to thank Rebecca Williamson and Sabrina Simoes for critical reading of the manuscript. We also acknowledge the expert technical help of Elizabeta Micevska. SIM and spinningdisk images were collected in the Confocal and Specialized Microscopy Shared Resource of the Herbert Irving Comprehensive Cancer Center at Columbia University, supported by NIH grant \#P30 CA013696 (National Cancer Institute) and we thank Theresa Swayne, Cedric Espenel and Laura Munteanu for their expert help. The work was supported by NIH grant R01 NS056049 to GDP. CM was the recipient of a post-doctoral fellowship from the Philippe Chatrier Foundation.

\section{Author contributions}

C.M. and G.D.P. conceived the research. C.M., H.T., J.Y., J.B., C.D.A., Y.Y.K., B.Z. and R.B.C. performed and analysed the experiments. H.T., J.Y., C.D.A., B.Z. and R.B.C. edited the manuscript. C.M. and G.D.P. wrote the manuscript.

\section{Additional information}

Supplementary Information accompanies this paper at http://www.nature.com/ naturecommunications

Competing financial interests: The authors declare no competing financial interests.

Reprints and permission information is available online at http://npg.nature.com/ reprintsandpermissions/

How to cite this article: Marquer, C. et al. Arf6 controls retromer traffic and intracellular cholesterol distribution via a phosphoinositide-based mechanism. Nat. Commun. 7:11919 doi: 10.1038/ncomms11919 (2016).

\section{(c) (i)}

This work is licensed under a Creative Commons Attribution 4.0 International License. The images or other third party material in this article are included in the article's Creative Commons license, unless indicated otherwise in the credit line; if the material is not included under the Creative Commons license, users will need to obtain permission from the license holder to reproduce the material. To view a copy of this license, visit http://creativecommons.org/licenses/by/4.0/ 